\title{
NONSMOOTH CRITICAL POINT THEORY AND NONLINEAR ELLIPTIC EQUATIONS AT RESONANCE
}

\author{
Nikolaos C. Kourogenis and Nikolaos S. Papageorgiou
}

\begin{abstract}
In this paper we complete two tasks. First we extend the nonsmooth critical point theory of Chang to the case where the energy functional satisfies only the weaker nonsmooth Ceramı condition and we also relax the boundary conditions. Then we study semilinear and quasilinear equations (involving the $p$-Laplacian). Using a variational approach we establish the existence of one and of multiple solutions. In the simple existence theorems, we allow the right hand side to be discontinuous. In that case in order to have an existence theory, we pass to a multivalued approximation of the orıginal problem by, roughly speakıng, filling in the gaps at the discontinuity points.
\end{abstract}

\section{Introduction}

The purpose of this paper is twofold. First, we want to extend the nonsmooth critical point theory of Chang [7], by replacing the compactness and the boundary conditions. Second, we want to study nonlinear elliptic problems at resonance and establish the existence of solutions and of multiple solutions.

Chang [7], in order to study equations with discontinuities, developed an extension of the classical smooth critical point theory, to nonsmooth locally Lipschitz functionals. The theory of Chang was based on the subdifferential of locally Lipschitz functionals due to Clarke [8]. Using this subdifferential, Chang proposed a generalization of the well-known "Palais-Smale condition" ((PS)condition) and through it obtained various minimax principles concerning the existence and characterization of critical points for locally Lipschitz functions. As is the case with the "smooth" theory, we can extend the theory of Chang in two directions. One is to weaken the (PS)-condition, and use a nonsmooth counterpart of the Cerami condition (C-condition; see Cerami [6]). It was shown by Bartolo-Benci-Fortunato [4], that in the smooth case, we can have a deformation theorem and through it minimax principles about critical points using

1991 Mathematics Subject Classification: 35J20, 35R70, 49F15, 58E05.

Key words and phrases: Nonsmooth critical point theory, locally Lipschitz function, subdifferential, linkıng, nonsmooth Palais-Smale condition, nonsmooth Ceramı condition, Mountain pass theorem, Saddle point theorem, problems at resonance, $p$-Laplacian, first eigenvalue.

Received April 7, 1999; revised September 20, 1999. 
only the weaker C-condition. The other possible generalization, is to relax the boundary conditions, namely allow certain inequalities in the minimax principles to be non-strict. Such generalizations are already well known in the context of the "smooth" theory (see for example Ghoussoub [9]). In this work we present extensions of the theory of Chang in both the aforementioned directions (see section 3).

The second task of this paper is to study nonlinear elliptic problems at resonance. In sections 4 and 5 we consider equations driven by the $p$-Laplacian operator $(p \geq 2)$ and in section 6 we deal with semilinear equations $(p=2)$. Moreover, in sections 4 and 6 , the right hand side nonlinearity $f(z, \cdot)$ is in general discontinuous. On the other hand, in section 5 the nonlinearity $f(z, \cdot)$ is continuous, but we prove the existence of at least two nontrivial solutions based on an abstract multiplicity result under splitting due to Brezis-Nirenberg [5]. In our work the resonance is simple, namely we have that the potential function $F(z, x)=\int_{0}^{x} f(z, r) d r$ goes to $\pm \infty$ as $|x| \rightarrow+\infty$. In this respect our work is similar to that of Ahmad-Lazer-Paul [3] and Rabinowitz [21, Theorem 4.12, p. 25]. Both works deal with semilinear equations and have continuous nonlinearities. Strongly resonant problems (i.e., $F(z, x)$ having finite limits as $x \rightarrow \pm \infty$ ), were studied by Thews [23], Bartolo-Benci-Fortunato [4], Ward [25] (for semilinear problems with continuous nonlinearity) and Kourogenis-Papageorgiou [17] (for quasilinear problems with discontinuous nonlinearity). Multiplicity results for semilinear resonant problems with continuous right hand side, were obtained by Solimini [22], Ahmad [2], Goncalves-Miyagaki [10], [11] and LandesmanRobinson-Rumbos [18]. For quasilinear problems involving the $p$-Laplacian, existence and multiplicity results were obtained by the authors in a series of papers Kourogenis-Papageorgiou [14], [15], [16], [17]. Our work here complements and partially extends these works. In particular Theorems 10 and 11, extend the existence result of Kourogenis-Papageorgiou [14], where the growth and asymptotic conditions on $f(z, \cdot)$ are more restrictive. Also, our Theorem 12 compared to the results of Ahmad-Lazer-Paul [3] and Rabinowitz [24], allows a more general growth condition on the nonlinearity $f$, which in the aforementioned works was assumed to be independent of $z \in Z$, continuous and bounded.

In the next section for the convenience of the reader, we recall some basic definitions and facts from the critical point theory (smooth and nonsmooth) and the Brezis-Nirenberg abstract multiplicity result. In section 3 we develop the extensions of the theory of Chang and finally in sections 4, 5 and 6 we study resonant elliptic problems. In sections 4 and 5 the equations are quasilinear involving the $p$-Laplacian, while in section 6 the problem is semilinear. Moreover, in sections 4 and 6 the nonlinearity is discontinuous and in section 5 we prove a multiplicity theorem.

\section{Mathematical preliminaries}

The nonsmooth critical point theory of Chang [7] is based on the subdifferential theory of locally Lipschitz functions due to Clarke [8]. So let $X$ be a 
Banach space and $X^{*}$ its topological dual. A function $\phi: X \rightarrow \boldsymbol{R}$ is said to be "locally Lipschitz", if for every $x \in X$, there exists a neighbourhood $U$ of $x$ and a constant $k>0$ depending on $U$ such that $|\phi(z)-\phi(y)| \leq k\|z-y\|$ for all $z, y \in U$. For such a function we define "generalized directional derivative" $\phi^{0}(x ; h)$ at $x \in X$ in the direction $h \in X$ by

$$
\phi^{0}(x ; h)=\varlimsup_{\substack{x^{\prime} \rightarrow x \\ \lambda \downarrow 0}} \frac{\phi\left(x^{\prime}+\lambda h\right)-\phi\left(x^{\prime}\right)}{\lambda} .
$$

The function $h \rightarrow \phi^{0}(x ; h)$ is sublinear, continuous. So by the Hahn-Banach theorem we know that $\phi^{0}(x ; \cdot)$ is the support function of a nonempty, convex and $w^{*}$-compact set

$$
\partial \phi(x)=\left\{x^{*} \in X^{*}:\left(x^{*}, h\right) \leq \phi^{0}(x ; h) \text { for all } h \in X\right\} .
$$

The set $\partial \phi(x)$ is called "the generalized or Clarke subdifferential" of $\phi$ at $x$. If $\phi, \psi: X \rightarrow \boldsymbol{R}$ are locally Lipschitz functions, then $\partial(\phi+\psi)(x) \subseteq \partial \phi(x)+\partial \psi(x)$, while for any $\lambda \in \boldsymbol{R}$ we have $\partial(\lambda \phi)(x)=\lambda \partial \phi(x)$. Moreover, if $\phi: X \rightarrow \boldsymbol{R}$ is also convex, then this subdifferential coincides with the subdifferential in the sense of convex analysis. If $\phi$ is strictly differentiable (in particular if $\phi \in C^{1}(X, \boldsymbol{R})$ ), then $\partial \phi(x)=\left\{\phi^{\prime}(x)\right\}$. A point $x \in X$ is a "critical point" of $\phi$ if $0 \in \partial \phi(x)$. For details and additional results we refer to the monograph of Clarke [8].

It is well known that the smooth critical point theory, uses a compactnesstype condition, known as the "Palais-Smale condition" (PS-condition for short). So if $\phi: X \rightarrow \boldsymbol{R}$ is a $C^{1}$ function and $c \in \boldsymbol{R}$, we say that $\phi$ satisfies the PalaisSmale condition at level $c$ (the (PS) $c$-condition for short), if for every sequence $\left\{x_{n}\right\}_{n \geq 1} \subseteq X$ such that $\phi\left(x_{n}\right) \stackrel{n \rightarrow \infty}{\longrightarrow} c$ and $\phi^{\prime}(x) \stackrel{n \rightarrow \infty}{\longrightarrow} 0$, has a strongly convergent subsequence. If this is true for every $c \in \boldsymbol{R}$, then we say that $\phi(\cdot)$ satisfies the $P S$-condition. In the nonsmooth setting with $\phi: X \rightarrow \boldsymbol{R}$ locally Lipschitz, this condition takes the form that every sequence $\left\{x_{n}\right\}_{n \geq 1} \subseteq X$ such that $\phi\left(x_{n}\right) \stackrel{n \rightarrow \infty}{\longrightarrow}$ $c$ and $m\left(x_{n}\right) \stackrel{n \rightarrow \infty}{\longrightarrow} 0$, has a strongly convergent subsequence. Here $m(x)=$ $\inf \left\{\left\|x^{*}\right\|: x^{*} \in \partial \phi(x)\right\}$ and the infimum is actually obtained, because $\partial \phi(x)$ is $w^{*}$ compact and the norm $\|\cdot\|$ is $w^{*}$-lower semicontinuous. If $\phi \in C^{1}(X, \boldsymbol{R})$, then since $\partial \phi(x)=\left\{\phi^{\prime}(x)\right\}$, we see that the above nonsmooth notion is an extension of the original smooth one.

A weaker form of the (PS)-condition was introduced by Cerami [6], who required that every sequence $\left\{x_{n}\right\}_{n>1} \subseteq X$ such that $\phi\left(x_{n}\right) \rightarrow c$ and $\left(1+\left\|x_{n}\right\|\right) \phi^{\prime}\left(x_{n}\right) \rightarrow$ 0 as $n \rightarrow \infty$, has a strongly convergent subsequence. It was proved by BartoloBenci-Fortunato [4], that this weaker condition suffices to have a deformation theorem and using that derive minimax principles. In the next section we do the same thing in the nonsmooth setting for the theory of Chang [7], using the nonsmooth version of Cerami's condition, which says that every sequence $\left\{x_{n}\right\}_{n \geq 1} \subseteq X$ such that $\phi\left(x_{n}\right) \stackrel{n \rightarrow \infty}{\longrightarrow} c$ and $\left(1+\left\|x_{n}\right\|\right) m\left(x_{n}\right) \stackrel{n \rightarrow \infty}{\longrightarrow} 0$, has a strongly convergent subsequence. In what follows we write $(\mathbf{C})_{c}$-condition (or simply C-condition if it holds for every level $c \in \boldsymbol{R}$ ), for the Cerami condition at level $c$. 
As we already mentioned in the introduction, our multiplicity theorem in section 5 will be based on an abstract multiplicity result in the presence of splitting due to Brezis-Nirenberg [5]. Here we recall the exact statement of this result:

THeOREM 1. If $X$ is a Banach space, $X=Y \oplus V$ with $\operatorname{dim} Y<\infty, R: X \rightarrow \boldsymbol{R}$ is a $C^{1}$-functional satisfying the Palals-Smale condition ((PS)-condition) such that for some $r>0$ the following hold

(i) $R(x) \geq 0$ for $x \in V,\|x\| \leq r$,

(ii) $R(x) \leq 0$ for $x \in Y,\|x\| \leq r$,

(iii) $R$ is bounded below and $\inf _{X} R<0$, then $R(\cdot)$ has at least two nonzero critical points.

Next consider the following nonlinear eigenvalue problem. Here $Z \subseteq \boldsymbol{R}^{N}$ is a bounded domain with a $C^{1}$-boundary $\Gamma$ :

$$
\left\{\begin{array}{l}
-\operatorname{div}\left(\|D x(z)\|^{p-2} D x(z)\right)=\lambda|x(z)|^{p-2} x(z) \text { a.e. on } Z \\
x_{\left.\right|_{\Gamma}}=0 .
\end{array}\right\}
$$

The least real number $\lambda$ for which (1) has a nontrivial solution is called the first eigenvalue of the negative $p$-Laplacian $-\Delta_{p} x=-\operatorname{div}\left(\|D x\|^{p-2} D x\right)$ with Dirichlet boundary conditions (i.e. $\left(-\Delta_{p}, W_{0}^{1, p}(Z)\right)$ ) and it is denoted by $\lambda_{1}$. This first eigenvalue $\lambda_{1}$ is positive, isolated and simple (i.e. the associated eigenfunctions are constant multiples of each other). Moreover, we have the following variational characterization of $\lambda_{1}>0$ via the Rayleigh quotient, namely

$$
\lambda_{1}=\min \left[\frac{\|D x\|_{p}^{p}}{\|x\|_{p}^{p}}: x \in W_{0}^{1, p}(Z), x \neq 0\right] .
$$

This minimum is realized at the normalized eigenfunction $u_{1}$ (recall that $\lambda_{1}$ is simple). Note that if $u_{1}$ minimizes the Rayleigh quotient, then so does $\left|u_{1}\right|$ and so we infer that the first eigenfunction $u_{1}$ does not change sign on $Z$. Moreover, we can show that $u_{1}(z) \neq 0$ a.e. on $Z$ and so we may assume that $u_{1}(z)>0$ a.e. on $Z$ (note that by the nonlinear elliptic regularity theory $u_{1} \in C_{\mathrm{loc}}^{1, \beta}(Z), 0<$ $\beta<1$; see Tolksdorf [24]). For details on these facts, we refer to Lindqvist [20].

The Ljusternik-Schnirelmann theory gives, in addition to $\lambda_{1}$, a whole strictly increasing sequence of positive numbers $\lambda_{1}<\lambda_{2}<\lambda_{3}<\cdots<\lambda_{k}<\cdots$ for which there exist nontrivial solutions for problem (1). In other words the spectrum $\sigma\left(-\Delta_{p}\right)$ of $\left(-\Delta_{p}, W_{0}^{1, p}(Z)\right)$ contains at least these points $\left\{\lambda_{k}\right\}_{k \geq 1}$. Nothing is known about the possible existence of other points in $\sigma\left(-\Delta_{p}\right) \subseteq\left[\lambda_{1}, \infty\right) \subseteq \boldsymbol{R}_{+}$. However, if $Y=\left\langle u_{1}\right\rangle=\boldsymbol{R} u_{1}$ and $V$ a topological complement (i.e., $W_{0}^{1, p}(Z)=$ $Y \oplus V)$, then because $\lambda_{1}$ is isolated, we have

$$
\lambda^{*}=\inf \left[\frac{\|D v\|_{p}^{p}}{\|v\|_{p}^{p}}: v \in V, v \neq 0\right]>\lambda_{1} .
$$

If $p=2$, then $\lambda^{*}=\lambda_{2}$ the second eigenvalue of $\left(-\Delta, W_{0}^{1, p}(Z)\right)$. 


\section{Abstract nonsmooth critical point theory}

In this section we extend Chang's theory to the case where the locally Lipschitz functional satisfies the nonsmooth C-condition and the boundary conditions are relaxed. So throughout this section $X$ is a reflexive Banach space and $\phi: X \rightarrow \boldsymbol{R}$ a locally Lipschitz functional. For each $c \in \boldsymbol{R}$ we set

$$
K_{c}=\{x \in X: 0 \in \partial \phi(x), \phi(x)=c\}
$$

and

$$
\phi^{c}=\{x \in X: \phi(x) \leq c\} .
$$

Recalling that $\operatorname{Gr} \partial \phi=\left\{\left(x, x^{*}\right) \in X \times X^{*}: x^{*} \in \partial \phi(x)\right\}$ is sequentially closed in $X \times X_{w}^{*}$ (here $X_{w}^{*}$ denotes the space $X^{*}$ furnished with the weak topology), we see at once that if $\phi(\cdot)$ satisfies the nonsmooth C-condition, then $K_{c}$ is compact. We start with two auxiliary results which are analogous to Lemmata 3.2 and 3.3 of Chang [7]. In what follows for $\delta>0,\left(K_{c}\right)_{\delta}=\left\{x \in X: d\left(x, K_{c}\right)<\delta\right\}$.

Lemma 2. If $\phi: X \rightarrow \boldsymbol{R}$ satisfies the nonsmooth $(\mathrm{C})_{c}$-condition, then for each $\delta>0$ there exist $\gamma>0$ and $0<\varepsilon$ such that

$$
(1+\|x\|) m(x) \geq \gamma \text { for all } x \in\left(K_{c}\right)_{\delta}^{c} \text { and } c-\varepsilon \leq \phi(x) \leq c+\varepsilon .
$$

Proof. Suppose not. Then for $\gamma_{n}, \varepsilon_{n} \downarrow 0$, we can find $x_{n} \in\left(K_{c}\right)_{\delta}^{c}, \phi\left(x_{n}\right) \rightarrow c$ such that $\left(1+\left\|x_{n}\right\|\right) m\left(x_{n}\right) \rightarrow 0$. By virtue of the nonsmooth $(\mathrm{C})_{c}$-condition, we may assume that $x_{n} \rightarrow x$ in $X$. Therefore we have $\phi(x)=c$. Moreover, from Chang [7, p. 105] we know that $m(x) \leq \underline{\lim } m\left(x_{n}\right)=0 \Rightarrow m(x)=0$ and so $x \in K_{c}$, a contradiction (recall that for any $\left.n \geq 1, x_{n} \in\left(K_{c}\right)_{\delta}^{c}\right)$. This proves the lemma.

Q.E.D.

The second lemma gives us a locally Lipschitz vector field which will play the role of a pseudo-gradient vector field of the smooth case.

Lemma 3. If $\phi: X \rightarrow \boldsymbol{R}$ satisfies the nonsmooth $(\mathrm{C})_{c}$-condition, $\delta>0$ is given and $\gamma, \varepsilon>0$ are as in Lemma 2, then there exists a locally Lipschitz vector field $v:\{x \in X:|\phi(x)-c| \leq \varepsilon\} \cap\left(K_{c}\right)_{\delta}^{c} \rightarrow X$ such that

$$
\|v(x)\| \leq(1+\|x\|)
$$

and

$$
\left(x^{*}, v(x)\right) \geq \frac{\gamma}{2} \text { for all } x^{*} \in \partial \phi(x)
$$

Proof. We follow the proof of Lemma 3.3 of Chang [7] with the necessary modifications.

Let $x \in X$ and let $x^{*} \in \partial \phi(x)$ such that $m(x)=\left\|x^{*}\right\|$. We have $B\left(0,\left\|x^{*}\right\|\right) \cap$ $\partial \phi(x)=\emptyset$ (where $\left.B\left(0,\left\|x^{*}\right\|\right)=\left\{z^{*} \in X^{*}:\left\|z^{*}\right\|<\left\|x^{*}\right\|\right\}\right)$. So by the separation 
theorem, we can find $u \in X$ with $\|u\|=1$ such that $\left(z^{*}, u\right) \leq\left(x^{*}, u\right) \leq\left(y^{*}, u\right)$ for all $z^{*} \in B\left(0,\left\|x^{*}\right\|\right)$ and all $y^{*} \in \partial \phi(x)$. Recall that $\sup \left[\left(z^{*}, u\right): z^{*} \in B\left(0,\left\|x^{*}\right\|\right)\right]=$ $\left\|x^{*}\right\|$. Hence we obtain $\gamma /(2(1+\|x\|))<\left\|x^{*}\right\| \leq\left(y^{*}, u\right)$ for all $y^{*} \in \partial \phi(x)$. Exploiting the fact that the multifunction $v \rightarrow \partial \phi(v)$ is upper semicontinuous from $X$ into $X_{w}^{*}$, for each $x \in\left\{x \in X:|\phi(x)-c| \leq \varepsilon, x \in\left(K_{c}\right)_{\delta}^{c}\right\}$ we can find $\theta>0$ such that for all $y \in B(x, \theta)=\{y \in X:\|y-x\|<\theta\}$ and all $y^{*} \in \partial \phi(y)$ we have $\gamma / 2(1+\|y\|)<\left(y^{*}, u\right)$. Then $\{B(x, \theta)\}$ is a cover of the set $\{x \in X$ : $\left.|\phi(x)-c| \leq \varepsilon, x \in\left(K_{c}\right)_{\delta}^{c}\right\}$. By paracompactness we can find a locally Lipschitz finite refinement $\left\{U_{i}\right\}_{l \in I}$. Let $\left\{\xi_{l}\right\}_{l \in I}$ be a locally Lipschitz partition of unity subordinate to $\left\{U_{i}\right\}_{l \in I}$ and let $v(x)=(1+\|x\|)_{i \in I} \xi_{i}(x) u_{i}$. Evidently $v:\{x \in X:$ $\left.|\phi(x)-c| \leq \varepsilon, x \in\left(K_{c}\right)_{\delta}^{c}\right\} \rightarrow X$ is locally Lipschitz and

$$
\|v(x)\| \leq(1+\|x\|)
$$

while

$$
\left(y^{*}, v(x)\right)=(1+\|x\|) \sum_{l \in I} \xi_{i}(x)\left(y^{*}, u_{i}\right)>\frac{\gamma}{2}
$$

The next theorem (deformation theorem) is the key tool for the nonsmooth critical point theory. It extends Theorem 3.1 of Chang [7].

THEOREM 4. If $\phi: X \rightarrow \boldsymbol{R}$ satisfies the nonsmooth $(\mathrm{C})_{c}$-condition, then for every $\varepsilon_{0}>0$, every neighborhood $U$ of $K_{c}$ (if $K_{c}=\emptyset$, we take $\left.U=\emptyset\right)$, there exist $0<\varepsilon<\varepsilon_{0}$ and $\eta:[0,1] \times X \rightarrow X$ continuous such that for all $(t, x) \in[0,1] \times X$ we have

(a) $\|\eta(t, x)-x\| \leq e(1+\|x\|) t$

(b) $|\phi(x)-c| \geq \varepsilon_{0} \Rightarrow \eta(t, x)=x$;

(c) $\eta\left(\{1\} \times \phi^{c+\varepsilon}\right) \subseteq \phi^{c-\varepsilon} \cup U$;

(d) $\phi(\eta(t, x)) \leq \phi(x)$;

(e) $\eta(t, x) \neq x \Rightarrow \phi(\eta(t, x))<\phi(x)$.

Proof. By virtue of the compactness of $K_{c}$, we can find $\delta>0$ such that $\left(K_{c}\right)_{3 \delta} \subseteq U$. By virtue of Lemma 2, we can find $\gamma>0$ and $0<\bar{\varepsilon}<\varepsilon_{0}$ such that $\gamma \leq(1+\|x\|) m(x)$ for all $x \in\left(K_{c}\right)_{\delta}^{c}$ and $c-\bar{\varepsilon} \leq \phi(x) \leq c+\bar{\varepsilon}$. Consider the following two closed sets in $X$ :

$$
C_{1}=\{x \in X:|\phi(x)-c| \geq \bar{\varepsilon}\} \cup \overline{\left(K_{c}\right)_{\delta}}
$$

and

$$
C_{2}=\left\{x \in X:|\phi(x)-c| \leq \frac{\bar{\varepsilon}}{2}\right\} \cap\left(K_{c}\right)_{2 \delta}^{c} .
$$

Evidently $C_{1} \cap C_{2}=\emptyset$ and so we can find $\xi: X \rightarrow[0,1]$ a locally Lipschitz function such that $\xi_{\left.\right|_{C_{1}}}=0$ and $\xi_{\left.\right|_{C_{2}}}=1$. Using the vector field $v(x)$ obtained in Lemma 3 , we define $L: X \rightarrow X$ by 


$$
L(x)= \begin{cases}-\xi(x) v(x) & \text { if }|\phi(x)-c| \leq \bar{\varepsilon} \text { and } x \in\left(K_{c}\right)_{\delta}^{c} \\ 0 & \text { otherwise. }\end{cases}
$$

Clearly $L(\cdot)$ is locally Lipschitz. Also we have for $x \in\{x \in X:|\phi(x)-c| \leq \bar{\varepsilon}$, $\left.x \in\left(K_{c}\right)_{\delta}^{c}\right\}:$

$$
\|L(x)\|=\xi(x)\|v(x)\| \leq(1+\|x\|)
$$

and

$$
\left(x^{*}, L(x)\right)=-\xi(x)\left(x^{*}, v(x)\right) \leq-\xi(x) \frac{\gamma}{2} .
$$

For every fixed $x \in X$, we consider the following Banach space-valued Cauchy problem:

$$
\left\{\begin{array}{l}
\frac{d}{d t} \eta(x ; t)=L(\eta(x ; t)) \text { a.e. on }[0,1] \\
\eta(x ; 0)=x
\end{array}\right\}
$$
have

Since $L$ is locally Lipschitz, problem $(6)$ has a unique solution $\eta(x ; \cdot)$. We

$$
\begin{aligned}
\|\eta(x ; t)-x\| & \leq \int_{0}^{t}\|L(\eta(x ; s))\| d s \\
& \leq \int_{0}^{t}(1+\|\eta(x ; s)\|) d s \quad(\text { see }(4)) \\
& \leq \int_{0}^{t}\|\eta(x ; s)-x\| d s+(1+\|x\|) t .
\end{aligned}
$$

By Gronwall's inequality, we have

$$
\|\eta(x ; t)-x\| \leq(1+\|x\|) t e,
$$

and so (a) is proved. Also if $|\phi(x)-c| \geq \bar{\varepsilon}$, then $\xi(x)=0$ and so $\eta(x ; t)=x$. So we have proved (b).

Next let $h(t)=\phi(\eta(x ; t))$. We know that $h:[0,1] \rightarrow X$ is locally Lipschitz, hence differentiable almost everywhere. Moreover, we have (see Chang [7, p. 106])

$$
\begin{aligned}
h^{\prime}(t) & \leq \max \left[\left(x^{*}, \frac{d}{d t} \eta(x ; t)\right): x^{*} \in \partial \phi(\eta(x ; t))\right] \quad \text { a.e. on } T \\
& =\max \left[\left(x^{*}, L(\eta(x ; t))\right): x^{*} \in \partial \phi(\eta(x ; t))\right] \text { a.e. on } T \\
\Rightarrow & h^{\prime}(t)= \begin{cases}-\xi(x) \frac{\gamma}{2} & \text { if }|\phi(x)-c| \leq \bar{\varepsilon} \text { and } x \in\left(K_{c}\right)_{\delta}^{c} \\
0 & \text { otherwise }\end{cases} \\
\Rightarrow & h(\cdot) \text { is nonincreasing. }
\end{aligned}
$$


Therefore we infer that for all $t \in T$ and all $x \in X, \phi(\eta(x ; t)) \leq \phi(x)$. This proves (d). Also if $|\phi(x)-c| \leq \bar{\varepsilon}$ and $x \in\left(K_{c}\right)_{\delta}^{c}$, we have

$$
\begin{gathered}
\phi(x)-\phi(\eta(x ; t))=-\int_{0}^{t} h^{\prime}(s) d s \geq \xi(x) \frac{\gamma}{2}>0 \\
\Rightarrow \phi(\eta(x ; t))<\phi(x) \quad \text { if } \eta(x ; t) \neq x, \quad \text { which proves (e). }
\end{gathered}
$$

It remains to show conclusion (c) of the theorem. Let $\rho>0$ such that $\left(\overline{K_{c}}\right)_{2 \delta}^{c} \subseteq B(0, \rho)$. Choose $0<\varepsilon \leq \bar{\varepsilon}$ such that

$$
4 \varepsilon \leq \gamma \quad \text { and } \quad 4 \varepsilon(1+\rho) e \leq \delta \gamma .
$$

We proceed by contradiction. Let $x \in \phi^{c+\varepsilon}$ and suppose that $\phi(\eta(x ; t))>$ $c-\varepsilon$ and $\eta(x ; 1) \in U^{c}$. We have

$$
c-\varepsilon<\phi(\eta(x ; t)) \leq c+\varepsilon \text { for all } t \in[0,1] .
$$

Also it can not happen that $\eta(\{x\} \times[0,1]) \cap\left(K_{c}\right)_{2 \delta}=\emptyset$. Indeed, if this intersection is empty, from (5) and the properties of $\xi(\cdot)$, we have

$$
\frac{\gamma}{2} \leq-\int_{0}^{1} h^{\prime}(s) d s=\phi(x)-\phi(\eta(x ; 1)) .
$$

But $x \in \phi^{c+\varepsilon}$. So combining this with (9), we obtain

$$
\begin{gathered}
\phi(x)-\phi(\eta(x ; 1))<2 \varepsilon \\
\Rightarrow \quad \gamma<4 \varepsilon
\end{gathered}
$$

which contradicts the choice of $\varepsilon>0$ (see (8)). Therefore we can find $0 \leq t_{1}<$ $t_{2} \leq 1$ such that

$$
d\left(\eta\left(x ; t_{1}\right), K_{c}\right)=2 \delta, \quad d\left(\eta\left(x ; t_{2}\right), K_{c}\right)=3 \delta
$$

and

$$
2 \delta<d\left(\eta(x ; t), K_{c}\right)<3 \delta \text { for all } t_{1}<t<t_{2} .
$$

Using once again (5), we have

$$
\begin{gathered}
\frac{\gamma}{2}\left(t_{2}-t_{1}\right) \leq \int_{t_{2}}^{t_{1}} h^{\prime}(s) d s=\phi\left(\eta\left(x ; t_{1}\right)\right)-\phi\left(\eta\left(x ; t_{2}\right)\right)<2 \varepsilon \\
\Rightarrow \quad t_{2}-t_{1}<\frac{4 \varepsilon}{\gamma} .
\end{gathered}
$$

Using this last inequality and arguing as in the proof of (a), we obtain

$$
\begin{aligned}
\delta & \leq\left\|\eta\left(x ; t_{2}\right)-\eta\left(x ; t_{1}\right)\right\| \leq \int_{t_{1}}^{t_{2}}\|L(\eta(x ; s))\| d s \\
& \leq e\left(1+\left\|\eta\left(x ; t_{1}\right)\right\|\right)\left(t_{2}-t_{1}\right)<(1+\rho) \frac{4 \varepsilon}{\gamma} e
\end{aligned}
$$

which contradicts the choice of $\varepsilon>0$ (see (8)). This proves (c) and so the proof of the theorem is complete.

Q.E.D. 
Using this theorem we can derive useful minimax principles for critical points in the nonsmooth setting. We start by introducing a basic notion of critical point theory.

Definition. Let $A, C \subseteq X$. We say that " $C$ links $A$ ", if $A \cap C=\emptyset$ and $C$ is not contractible in $X \backslash A$.

Remark. It is a well-known consequence of degree theory, that if $X$ is finite dimensional and $U$ is an open bounded neighborhood of $x$, then $\partial U$ ( $=$ the boundary of $U$ ) is not contractible in $X \backslash\{x\}$.

The next abstract minimax principle, will generate as byproducts the nonsmooth "Mountain Pass Theorem", "Saddle Point Theorem" and "Linking Theorem", under the nonsmooth C-condition.

Theorem 5. If $A, C \subseteq X$ are nonempty, $A$ is closed, $C$ links $A, \Gamma_{C}$ is the set of all contractions of $C, \phi: X \rightarrow \boldsymbol{R}$ satisfies the nonsmooth $(\mathrm{C})_{c}$-condition with

$$
c=\inf _{h \in \Gamma_{C}} \sup _{[0,1] \times C} \phi \circ h<\infty \text { and } \sup _{C} \phi \leq \inf _{A} \phi
$$

then $c \geq \inf _{A} \phi$ and $c$ is a critical value of $\phi . \quad$ Moreover, if $c=\inf _{A} \phi$, then there exists $x \in A$ such that $x \in K_{c}$.

Proof. Since by hypothesis $C$ links $A$, for every $h \in \Gamma_{C}$ we have $h([0,1] \times C)$ $\cap A \neq \emptyset$. So we infer that $c \geq \inf _{A} \phi$.

First we assume that $\inf _{A} \phi<c$. Suppose that $K_{c}=\emptyset$. Let $U=\emptyset$ and let $\varepsilon>0$ and $\eta:[0,1] \times X \rightarrow X$ be as in Theorem 4. Also from the definition of $c$, we can find $h \in \Gamma_{C}$ such that

$$
\phi(h(t, x)) \leq c+\varepsilon \text { for all } t \in[0,1], x \in C .
$$

Let $H:[0,1] \times C \rightarrow X$ be defined by

$$
H(t, x)= \begin{cases}\eta(2 t, x) & \text { if } 0 \leq t \leq \frac{1}{2} \\ \eta(1, h(2 t-1, x)) & \text { if } \frac{1}{2} \leq t \leq 1\end{cases}
$$

It is easy to check that $H \in \Gamma_{C}$ and for every $x \in C$ we have

$\phi(H(t, x))=\phi(\eta(2 t, x)) \leq \phi(x) \leq \sup _{C} \phi<c \quad$ for $0 \leq t \leq \frac{1}{2} \quad$ (see Theorem $\left.4(\mathrm{~d})\right)$

and $\phi(H(t, x))=\phi(\eta(1, h(2 t-1, x))) \leq c-\varepsilon<c$ for $1 / 2 \leq t \leq 1$ (see Theorem 4(c) and recall that $h(t, x) \in \phi^{c+\varepsilon}$ for all $t \in[0,1], x \in C$; see $\left.(10)\right)$. So we have contradicted the definition of $c$. This proves the nonemptiness of $K_{c}$ when $c>$ $\inf _{A} \phi$. Next assume that $c=\inf _{A} \phi$. We need to show that $K_{c} \cap A \neq \emptyset$. Sup- 
pose the contrary and let $U$ be a neighborhood of $K_{c}$ with $U \cap A=\emptyset$. Let $\varepsilon>0$ and $\eta:[0,1] \times X \rightarrow X$ be as in Theorem 4. As before let $h \in \Gamma_{C}$ such that $\phi(h(t, x)) \leq c+\varepsilon$ for all $(t, x) \in[0,1] \times C$. Then we define $H:[0,1] \times C \rightarrow X$ by

$$
H(t, x)= \begin{cases}\eta(2 t, x) & \text { if } 0 \leq t \leq \frac{1}{2} \\ \eta(1, h(2 t-1, x)) & \text { if } \frac{1}{2} \leq t \leq 1 .\end{cases}
$$

Again we can easily verify that $H \in \Gamma_{C}$. From Theorem 4, we know that for all $0 \leq t \leq 1 / 2$ and all $x \in C$, we have

$$
\begin{gathered}
\eta(2 t, x)=x \text { or } \phi(\eta(2 t, x))<\phi(x) \leq \inf _{A} \phi=c \\
\Rightarrow \quad \eta(2 t, x) \in A^{c} \quad \text { for all } 0 \leq t \leq \frac{1}{2} \text { and all } x \in C .
\end{gathered}
$$

For all $1 / 2 \leq t \leq 1$ and all $x \in C$, we have from Theorem $4(\mathrm{c})$

$$
\eta(1, h(2 t-1, x)) \subseteq \phi^{c-\varepsilon} \cup U
$$

while

$$
\left(\phi^{c-\varepsilon} \cup U\right) \cap A=\emptyset .
$$

So $H$ is a contraction of $C$ in $X \backslash A$, a contradiction. This proves the theorem.

Q.E.D.

As a first consequence of this minimax theorem, we derive an extended version of the nonsmooth "Mountain Pass Theorem" (see Chang [7, Theorem $3.4])$.

THEOREM 6. If there exist $x_{1} \in X$ and $r>0$ such that $\left\|x_{1}\right\|>r$, $\max \left[\phi(0), \phi\left(x_{1}\right)\right] \leq \inf [\phi(x):\|x\|=r]$ and $\phi: X \rightarrow \boldsymbol{R}$ satusfies the nonsmooth $(\mathrm{C})_{c}-$ condition with $c=\inf _{\gamma \in \Gamma} \max _{t \in[0,1]} \phi(\gamma(t))$ where $\Gamma=\{\gamma \in C([0,1], X): \gamma(0)=0$, $\left.\gamma(1)=x_{1}\right\}$ then $c \geq \inf [\phi(x):\|x\|=r]$ and $c$ is a critical value of $\phi$. Moreover, if $c=\inf [\phi(x):\|x\|=r]$, there exists a critical point $x$ of $\phi$ with $\phi(x)=c$ and $\|x\|=r$.

Proof. We will apply Theorem 5 with $A=\{x \in X:\|x\|=r\}$ and $C=\left\{0, x_{1}\right\}$. Clearly $C$ links $A$ and $c<\infty$. Let $\gamma \in \Gamma$ and define

$$
h(t, x)= \begin{cases}\gamma(t) & \text { if } x=0 \\ x_{1} & \text { if } x=x_{1} .\end{cases}
$$

Then $h \in \Gamma_{C}$ (see Theorem 5). So

$$
\inf _{\bar{h} \in \Gamma_{C}} \sup _{[0,1] \times C} \phi(\bar{h}(t, x)) \leq \phi(h(t, x)) \leq c .
$$


On the other hand, if $h \in \Gamma_{C}$, then

$$
\gamma(t)= \begin{cases}h(2 t, 0) & \text { if } 0 \leq t \leq \frac{1}{2} \\ h\left(2-2 t, x_{1}\right) & \text { if } \frac{1}{2} \leq t \leq 1\end{cases}
$$

belongs to $\Gamma$ and so

$$
\inf _{h \in \Gamma_{C}} \sup _{[0,1] \times C} \phi(h(t, x)) \geq c .
$$

From (11) and (12), we have

$$
c=\inf _{h \in \Gamma_{C}} \sup _{[0,1] \times C} \phi(h(t, x))
$$

and so we can apply Theorem 5 and finish the proof.

Q.E.D.

Remark. In addition to assuming the weaker nonsmooth $(\mathrm{C})_{c}$-condition (while Chang [7] assumes that $\phi$ satisfies the nonsmooth PS-condition), here we have proved the nonsmooth mountain pass theorem under relaxed boundary conditions, i.e. it can happen that $\max \left[\phi(0), \phi\left(x_{1}\right)\right]=\inf [\phi(x):\|x\|=r]$ (in Chang [7] the left hand side is strictly smaller than the right hand side). Also the choice of 0 as the second point in $C$ was done only for convenience. In fact we can replace 0 by any $x_{2} \in X$, provided that the hypothesis $\left\|x_{1}\right\|>r$ is replaced by the condition $\left\|x_{2}-x_{1}\right\|>r$.

The next important consequence of Theorem 5, is an extended version of the nonsmooth "Saddle Point Theorem" (see Chang [7, Theorem 3.3]).

THEOREM 7. If $X=Y \oplus V$, with $\operatorname{dim} Y<\infty$, there exists $r>0$ such that

$$
\max [\phi(x): x \in Y,\|x\|=r] \leq \inf [\phi(x): x \in V]
$$

and $\phi: X \rightarrow \boldsymbol{R}$ satisfies the nonsmooth $(\mathrm{C})_{c}$-condition where $c=$ $\inf _{\gamma \in \Gamma} \max _{x \in E} \phi(\gamma(x))$ with $\Gamma=\left\{\gamma \in C(E, X): \gamma_{\left.\right|_{\partial E}}=\right.$ identity $\}, E=\{x \in Y:\|x\| \leq r\}$ and $\partial E=\{x \in Y:\|x\|=r\}$, then $c \geq \inf _{V} \phi$ and $c$ is a critical value of $\phi$. Moreover, if $c=\inf _{V} \phi$, then

$$
V \cap K_{c} \neq \emptyset
$$

Proof. In this case we will apply Theorem 5 with $A=V$ and $C=\partial E$. Clearly from the compactness of $E$ (recall that by hypothesis $Y$ is finite dimensional), we have that $c<\infty$. Let $P: X \rightarrow Y$ be the projection operator (see $\mathrm{Hu}$ Papageorgiou [12, Propositions IV.7.8 and IV.7.9, pp. 502-503]). First we show that with the aforementioned choices, $C$ links $A$. Suppose not and let $h$ be a contraction of $C$ in $X \backslash V$. Let $H(t, x)=P h(t, x)$, which is a contraction of $C$ in $Y \backslash\{0\}$, a contradiction (see the remark following the definition of linking). So indeed $C$ links $A$. 

have

Next let $\gamma \in \Gamma$ and define $h(t, x)=\gamma((1-t) x)$. Evidently $h \in \Gamma_{C}$. So we

$$
\inf _{h \in \Gamma_{C}} \sup _{[0,1] \times C} \phi(h(t, x)) \leq \phi(h(t, x)) \leq c .
$$

Also if $h \in \Gamma_{C}$ and $h(1, x)=z_{1}$ for all $x \in C$, then we define

$$
\xi(t, x)= \begin{cases}h(t, x) & \text { if }(t, x) \in[0,1] \times C \\ z_{1} & \text { if }(t, x) \in\{1\} \times E\end{cases}
$$

which is continuous from $([0,1] \times C) \cup(\{1\} \times E)$ into $X$. Let $\theta: E \rightarrow$ $([0,1] \times C) \cup(\{1\} \times E)$ be a homeomorphism such that $\theta(C)=\{0\} \times C$. Then we see that $\xi \circ \theta \in \Gamma$ and so

$$
c \leq \inf _{h \in \Gamma_{C}} \sup _{[0,1] \times C} \phi(h(t, x)) .
$$

From (13) and (14) it follows that $c=\inf _{h \in \Gamma_{C}} \sup _{[0,1] \times C} \phi(h(t, x))$ and so we can apply Theorem 5 and finish the proof.

Q.E.D.

Remark. In this theorem too in addition to assuming a weaker compactness condition that Chang [7] (namely the nonsmooth $(\mathrm{C})_{c}$-condition), we also use a relaxed boundary condition, namely we do not require that $\sup [\phi(y): y \in Y$, $\|y\|=r]$ be strictly smaller than $\inf _{V} \phi$. In our formulation equality is also possible.

The next theorem is not in Chang [7] and is a nonsmooth generalization of the well-known "Linking Theorem" of Rabinowitz [21, Theorem 5.3, p. 28] with relaxed boundary condition.

TheOREM 8. If $X=Y \oplus V$ with $\operatorname{dim} Y<\infty$, with $0<r<R$ and $e \in V$ with $\|e\|=1$ such that

$$
\max [\phi(x): x \in \partial Q] \leq \inf [\phi(x): x \in \partial B(0, r) \cap V]
$$

where $Q=\{x=y+t e: y \in Y, t \geq 0,\|x\| \leq R\}$ and $\partial Q$ is its boundary in $Y \oplus \boldsymbol{R}$, and $\phi: X \rightarrow \boldsymbol{R}$ satisfies the nonsmooth $(\mathrm{C})_{c}$-condition where $c=$ $\inf _{\gamma \in \Gamma} \max _{x \in Q} \phi(\gamma(x))$ with $\Gamma=\left\{\gamma \in C(Q, X): \gamma_{l_{\partial Q}}=\right.$ identity $\}$, then $c \geq \inf [\phi(x)$ : $x \in \partial B(0, r) \cap V]$ and $c$ is a critical value. Moreover, if $c=\inf [\phi(x)$ : $x \in \partial B(0, r) \cap V]$, then $K_{c} \cap(\partial B(0, r) \cap V) \neq \emptyset$.

Proof. Because $Q$ is compact, it is clear that $c<\infty$. Let $P_{1}: X \rightarrow Y$ and $P_{2}: X \rightarrow V$ be the projection operators on $Y$ and $V$ and let $A=\partial B(0, r) \cap V$ and $C=\partial Q$. If $h(t, x)$ is a contraction of $C$ in $X \backslash A$, then $H(t, x)=P_{1} h(t, x)+$ $\left\|P_{2} h(t, x)\right\| e$ is a contraction of $C$ in $(V \oplus \boldsymbol{R} e) \backslash\{r e\}$ which is not possible (see the remark following the definition of linking). Moreover, as in the proof of Theorem 7, we can verify that $c=\inf _{h \in \Gamma_{C}} \sup _{[0,1] \times C} \phi \circ h$. So we can apply Theorem 5 to finish the proof.

Q.E.D. 
We conclude this section with a result which is a direct consequence of Corallary 2.3 of Zhong [26] and extends Theorem 3.5 of Chang [7].

THEOREM 9. If $\phi: X \rightarrow \boldsymbol{R}$ satisfies the nonsmooth $\mathrm{C}$-condition and is bounded below, then there exists $\hat{x} \in X$ such that $\phi(\hat{x})=\inf _{X} \phi=c$ (and so $\hat{x} \in K_{c}$ ).

\section{Equations at resonance with discontinuities}

Let $Z \in \boldsymbol{R}^{N}$ be a bounded domain with a $C^{1+\alpha}$-boundary $\Gamma(0<\alpha<1)$. We consider the following quasilinear elliptic resonant problem:

$$
\left\{\begin{array}{l}
-\operatorname{div}\left(\|D x(z)\|^{p-2} D x(z)\right)-\lambda_{1}|x(z)|^{p-2} x(z)=f(z, x(z)) \text { a.e. on } Z \\
x_{\left.\right|_{\Gamma}}=0,2 \leq p<\infty .
\end{array}\right\}
$$

We do not assume that $f(z, \cdot)$ is continuous. It is well-known then that (15) need not have a solution. Then we replace (15) by a multivalued equation which approximates it and is obtained by, roughly speaking, filling in the gaps at the discontinuity points of $f(z, \cdot)$. For the resulting elliptic inclusion, we can develop an existence theory based on the abstract results of section 3 . So we introduce the following two functions:

$$
f_{1}(z, x)=\varliminf_{x^{\prime} \rightarrow x} f\left(z, x^{\prime}\right)=\lim _{\delta \downarrow 0} \operatorname{essinf}_{\left|x^{\prime}-x\right|<\delta} f\left(z, x^{\prime}\right)
$$

and

$$
f_{2}(z, x)=\varlimsup_{x^{\prime} \rightarrow x} f\left(z, x^{\prime}\right)=\lim _{\delta \downarrow 0} \operatorname{ess} \sup _{\left|x^{\prime}-x\right|<\delta} f\left(z, x^{\prime}\right) .
$$

Let $\hat{f}(z, x)=\left\{y \in \boldsymbol{R}: f_{1}(z, x) \leq y \leq f_{2}(z, x)\right\}$ and consider the following multivalued approximation of (15):

$$
\left\{\begin{array}{l}
-\operatorname{div}\left(\|D x(z)\|^{p-2} D x(z)\right)-\lambda_{1}|x(z)|^{p-2} x(z) \in \hat{f}(z, x(z)) \text { a.e. on } Z \\
x_{\left.\right|_{\Gamma}}=0,2 \leq p<\infty .
\end{array}\right\}
$$

In the sequel we will be dealing with (16). By a solution of (16), we mean a function $x \in W_{0}^{1, p}(Z)$ such that

$$
\operatorname{div}\left(\|D x(\cdot)\|^{p-2} D x(\cdot)\right) \in L^{1}(Z)
$$

and

$$
-\operatorname{div}\left(\|D x(z)\|^{p-2} D x(z)\right)-\lambda_{1}|x(z)|^{p-2} x(z)=u(z) \text { a.e. on } Z,
$$

with $u \in L^{1}(Z)$ and

$$
f_{1}(z, x(z)) \leq u(z) \leq f_{2}(z, x(z)) \text { a.e. on } Z .
$$

In this section we prove an existence theorem for problem (16) under general growth conditions on the discontinuous nonlinearity $f(z, x)$, extending this way 
an earlier existence theorem by the authors [14]. The precise hypotheses on $f(z, x)$ are the following:

$\boldsymbol{H}(\boldsymbol{f})_{1}: \quad f: Z \times \boldsymbol{R} \rightarrow \boldsymbol{R}$ is a Borel measurable function such that

(i) $f_{1}, f_{2}$ are both $N$-measurable functions (i.e. for every $x: Z \rightarrow \boldsymbol{R}$ measurable function, $z \rightarrow f_{i}(z, x(z)), i=1,2$, are measurable); $x \in \boldsymbol{R}$

(ii) there exist $a_{1} \in L^{\infty}(Z)$ and $c_{1}>0$ such that for almost all $z \in Z$ and all

$$
|f(z, x)| \leq a_{1}(z)+c_{1}|x|^{p-1}
$$

(iii) for some $0<\mu<p$ we have $\underline{\lim }_{|x| \rightarrow \infty}(f(z, x) x-p F(z, x)) /|x|^{\mu}>0$ uniformly for almost all $z \in Z$ (recall that $F(z, x)=\int_{0}^{x} f(z, r) d r$, the potential function corresponding to $f$ );

(iv) $\varlimsup_{x \rightarrow 0}(p F(z, x)) /|x|^{p} \leq-\lambda_{1}$ uniformly for almost all $z \in Z$;

(v) there exists $\xi \neq 0$ such that $\int_{Z} F\left(z, \xi u_{1}(z)\right) d z \geq 0$.

Remark. Hypothesis $H(f)_{1}$ (iii) is a generalization of the well-known Ambrosetti-Rabinowitz condition introduced to deal with semilinear (i.e., $p=2$ ), superquadratic problems (see Rabinowitz [21, p. 9]). Indeed according to that condition $0<k F(z, x) \leq x f(z, x)$ for almost all $z \in Z$, all $|x| \geq M$ and for some $k>2$. This implies that $F(z, x) \geq \beta_{1}|x|^{k}-\beta_{2}$ a.e. on $Z$ for some $\beta_{1}, \beta_{2}>0$ (see Rabinowitz [21, Remark 2.13, p. 9]). Hence we have $(f(z, x) x-2 F(z, x)) /$ $|x|^{\mu} \geq(k-2)\left(F(z, x) /|x|^{\mu}\right) \geq(k-2) \beta_{1}|x|^{k-\mu}-(k-2) \beta_{2}\left(1 /|x|^{\mu}\right)$. So hypothesis $H(f)_{1}$ (iii) is satisfied provided $\mu<k$. Hypothesis $H(f)($ iv) is needed in order to get the linking necessary to apply the "Mountain Pass Theorem" (Theorem 6). Analogous conditions were used by Rabinowitz [21] (for the nonresonant problem, see hypothesis $\left(p_{3}\right)$, p. 9) and by Goncalves-Miyagaki [10], [11] (hypotheses $\left(g_{2}\right)$, $\left(g_{4}\right)$ in $[10]$ and hypothesis $(H)_{0}$ in [11]).

We have the following existence result.

THEOREM 10. If hypotheses $H(f)_{1}$ hold, then problem (16) has at least one nontrivial solution.

Proof. Let $\phi: W_{0}^{1, p}(Z) \rightarrow \boldsymbol{R}$ be defined as

$$
\phi(x)=\frac{1}{p}\|D x\|_{p}^{p}-\frac{\lambda_{1}}{p}\|x\|_{p}^{p}-\int_{Z} F(z, x(z)) d z .
$$

We know that $\phi(\cdot)$ is locally Lipschitz (see Chang [7, p. 111]). In what follows let $\psi: W_{0}^{1, p}(Z) \rightarrow \boldsymbol{R}$ be defined by $\psi(x)=\int_{Z} F(z, x(z)) d z$.

Claim 1. $\phi(\cdot)$ sattsfies the nonsmooth C-condition.

Let $\left\{x_{n}\right\}_{n \geq 1} \subseteq W_{0}^{1, p}(Z)$ be a sequence such that $\left|\phi\left(x_{n}\right)\right| \leq M$ for all $n \geq 1$ and $\left(1+\left\|x_{n}\right\|\right) m\left(x_{n}\right) \stackrel{n \rightarrow \infty}{\longrightarrow} 0$. Let $x_{n}^{*} \in \partial \phi\left(x_{n}\right)$ such that $m\left(x_{n}\right)=\left\|x_{n}^{*}\right\|, n \geq 1$, we 
know that

$$
x_{n}^{*}=A\left(x_{n}\right)-\lambda_{1}\left|x_{n}\right|^{p-2} x_{n}-u_{n}
$$

with $A: W_{0}^{1, p}(Z) \rightarrow W^{-1, q}(Z)$ the nonlinear operator defined by

$$
\langle A(x), y\rangle=\int_{Z}\|D x(z)\|^{p-2}(D x(z), D y(z))_{R^{N}} d z
$$

for all $y \in W_{0}^{1, p}(Z)$ and $u_{n} \in \partial \psi\left(x_{n}\right), n \geq 1$. From Chang [7] we know that $u_{n} \in$ $L^{q}(Z)$ and $f_{1}\left(z, x_{n}(z)\right) \leq u_{n}(z) \leq f_{2}\left(z, x_{n}(z)\right)$ a.e. on $Z$. We have

$$
\begin{aligned}
& \left|\left\langle x_{n}^{*}, x_{n}\right\rangle\right| \leq \varepsilon_{n} \text { and }\left|\phi\left(x_{n}\right)\right| \leq M, \quad n \geq 1, \quad \text { with } \varepsilon_{n} \downarrow 0, \\
& \Rightarrow \quad-\varepsilon_{n} \leq-\left\|D x_{n}\right\|_{p}^{p}+\lambda_{1}\left\|x_{n}\right\|_{p}^{p}+\int_{Z} u_{n}(z) x_{n}(z) d z \leq \varepsilon_{n}
\end{aligned}
$$

and

$$
-p M \leq\left\|D x_{n}\right\|_{p}^{p}-\lambda_{1}\left\|x_{n}\right\|_{p}^{p}-\int_{Z} p F(z, x(z)) d z \leq p M .
$$

Adding (17) and (18), we obtain

$$
-\varepsilon_{n}-p M \leq \int_{Z}\left(u_{n}(z) x_{n}(z)-p F\left(z, x_{n}(z)\right)\right) d z \leq \varepsilon_{n}+p M .
$$

By virtue of hypothesis $H(f)_{1}$ (iii), given $\varepsilon>0$, we can find $M_{1}=M_{1}(\varepsilon)>0$ such that for almost all $z \in Z$ and all $|x| \geq M_{1}$, we have

$$
f(z, x) x-p F(z, x) \geq(\beta-\varepsilon)|x|^{\mu}, \quad(\beta-\varepsilon>0) .
$$

On the other hand by virtue of hypothesis $H(f)_{1}$ (ii), for almost all $z \in Z$ and all $|x| \leq M_{1}$ we have

$$
|f(z, x) x-p F(z, x)| \leq a_{2}(z) \text { with } a_{2} \in L^{\infty}(Z) .
$$

Therefore from (20) and (21) we have that for almost all $z \in Z$ and all $z \in \boldsymbol{R}$

$$
f(z, x) x-p F(z, x) \geq(\beta-\varepsilon)|x|^{\mu}-\hat{a}_{2}(z), \quad \hat{a}_{2} \in L^{\infty}(Z) .
$$

Thus going back to inequality (19), we obtain

$$
\begin{aligned}
& (\beta-\varepsilon)\left\|x_{n}\right\|_{\mu}^{\mu} \leq \beta_{1} \text { for some } \beta_{1}>0 \\
\Rightarrow \quad & \left\{x_{n}\right\}_{n \geq 1} \subseteq L^{\mu}(Z) \text { is bounded. }
\end{aligned}
$$

Next from hypothesis $H(f)_{1}$ (ii), we see that for almost all $z \in Z$ and all $x \in \boldsymbol{R}$ we have

$$
F(z, x) \leq a_{3}(z)+\eta|x|^{q} \quad \text { with } a_{3} \in L^{\infty}(Z), \eta>0
$$

with $p<q<\min \left[p^{*}, p((N+\mu) / N), \mu+p\right]$. Since $\mu<q<p^{*}$, we can find $0<$ 
$\theta<1$ such that $1 / q=((1-\theta) / \mu)+\left(\theta / p^{*}\right)$. Using the interpolation inequality, we have

$$
\left\|x_{n}\right\|_{q} \leq\left\|x_{n}\right\|_{\mu}^{1-\theta}\left\|x_{n}\right\|_{p^{*}}^{\theta} \leq \beta_{2}\left\|x_{n}\right\|_{p^{*}}^{\theta} \leq \beta_{3}\left\|x_{n}\right\|_{1, p}^{\theta} \text { for some } \beta_{2}, \beta_{3}>0
$$

(recall that $W_{0}^{1, p}(Z)$ is embedded continuously in $L^{p^{*}}(Z)$ ). Recall from the choice of the sequence $\left\{x_{n}\right\}_{n \geq 1} \subseteq W_{0}^{1, p}(Z)$, that

$$
\begin{aligned}
& \left|\phi\left(x_{n}\right)\right| \leq M \text { for all } n \geq 1, \\
\Rightarrow & \frac{1}{p}\left\|D x_{n}\right\|_{p}^{p}-\frac{\lambda_{1}}{p}\left\|x_{n}\right\|_{p}^{p}-\int_{Z} F\left(z, x_{n}(z)\right) d z \leq M \\
\Rightarrow & \frac{1}{p}\left\|D x_{n}\right\|_{p}^{p} \leq \frac{\lambda_{1}}{p}\left\|x_{n}\right\|_{p}^{p}+\left\|a_{3}\right\|_{1}+\eta\left\|x_{n}\right\|_{q}^{q}+M \\
\leq & \frac{\lambda_{1} \beta_{4}}{p}\left\|x_{n}\right\|_{q}^{p}+\left\|a_{3}\right\|_{1}+\eta\left\|x_{n}\right\|_{q}^{q}+M \text { for some } \beta_{4}>0 .
\end{aligned}
$$

Let $r=q / p>1, r^{\prime}>1$ the conjugate exponent (i.e., $\left.(1 / r)+\left(1 / r^{\prime}\right)=1\right)$ and on $\left(\lambda_{1} \beta_{4} / p\right)\left\|x_{n}\right\|_{q}^{p}$ apply Young's inequality with $r, r^{\prime}>1$. We have

$$
\frac{\lambda_{1} \beta_{4}}{p}\left\|x_{n}\right\|_{q}^{p} \leq\left(\frac{\lambda_{1} \beta_{4}}{p}\right)^{r^{\prime}} \frac{1}{r^{\prime}}+\frac{p}{q}\left\|x_{n}\right\|_{q}^{q}
$$

Thus we can write that

$$
\begin{aligned}
\frac{1}{p}\left\|D x_{n}\right\| & \leq \beta_{5}\left(\lambda_{1}\right)+\beta_{6}\left\|x_{n}\right\|_{q}^{q} \text { for some } \beta_{5}\left(\lambda_{1}\right), \quad \beta_{6}>0 \\
& \leq \beta_{5}\left(\lambda_{1}\right)+\beta_{7}\left\|x_{n}\right\|^{\theta q} \text { for some } \beta_{7}>0 \quad(\text { see }(22))
\end{aligned}
$$

If $p<N$, then

$$
\begin{aligned}
\frac{1}{q} & =\frac{1-\theta}{\mu}+\frac{\theta}{p^{*}} \\
& \Rightarrow \theta q\left(\mu-p^{*}\right)=\mu p^{*}-q p^{*} \\
& \Rightarrow \theta q=\left(\mu p^{*}-q p^{*}\right) /\left(\mu-p^{*}\right)=p^{*}(q-\mu) /\left(p^{*}-\mu\right)<\mu<p
\end{aligned}
$$

because $q<(p(N+\mu)) / N \Rightarrow((q-p) N) / p<\mu$.

If $p \geq N$, then $p^{*}=+\infty$ (Sobolev embedding theorem) and so

$$
\frac{1}{q}=\frac{1-\theta}{\mu} \Rightarrow q-q \theta=\mu \Rightarrow \theta q=q-\mu<q-(q-p)=p
$$

because $q<\mu+p$.

Therefore in both cases we have $\theta q<p$. Using this fact in (23), together with Poincaré's inequality we infer that $\left\{x_{n}\right\}_{n \geq 1} \subseteq W_{0}^{1, p}(Z)$ is bounded. Thus by passing to a subsequence if necessary, we may assume that $x_{n} \stackrel{w}{\rightarrow} x$ in $W_{0}^{1, p}(Z)$ as $n \rightarrow \infty$. If we denote by $\langle\cdot, \cdot\rangle$ the duality brackets for the pair $\left(W_{0}^{1, p}(Z)\right.$, 
$\left.W^{-1, q}(Z)\right)$, we have

$$
\begin{gathered}
\left\langle A\left(x_{n}\right), x_{n}-x\right\rangle-\lambda_{1} \int_{Z}\left|x_{n}(z)\right|^{p-2} x_{n}(z)\left(x_{n}-x\right)(z) d z \\
-\int_{Z} u_{n}(z)\left(x_{n}-x\right)(z) d z \leq \varepsilon_{n}\left\|x_{n}-x\right\|_{1, p}
\end{gathered}
$$

with $\varepsilon_{n} \downarrow 0$. Since $W_{0}^{1, p}(Z)$ is embedded compactly in $L^{p}(Z)$ (Sobolev embedding theorem), we have that $x_{n} \stackrel{n \rightarrow \infty}{\longrightarrow} x$ in $L^{p}(Z)$ and so

$$
\int_{Z}\left|x_{n}(z)\right|^{p-2} x_{n}(z)\left(x_{n}-x\right)(z) d z \stackrel{n \rightarrow \infty}{\longrightarrow} 0
$$

and

$$
\int_{Z} u_{n}(z)\left(x_{n}-x\right)(z) d z \stackrel{n \rightarrow \infty}{\longrightarrow} 0
$$

Therefore we obtain

$$
\varlimsup \overline{\lim }\left\langle A\left(x_{n}\right), x_{n}-x\right\rangle \leq 0 .
$$

But we know (see for example Kourogenis-Papageorgiou [14]) that $A$ is monotone, demicontinuous, hence maximal monotone and of course pseudomonotone (see Hu-Papageorgiou [12]). Thus we have

$$
\begin{aligned}
&\left\langle A\left(x_{n}\right), x_{n}\right\rangle \rightarrow\langle A(x), x\rangle \\
& \Rightarrow \quad\left\|D x_{n}\right\|_{p} \rightarrow\|D x\|_{p} .
\end{aligned}
$$

We already know that $D x_{n} \stackrel{w}{\rightarrow} D x$ in $L^{p}\left(Z, \boldsymbol{R}^{N}\right)$. Since $L^{p}\left(Z, \boldsymbol{R}^{N}\right)$ is uniformly convex, we infer that $D x_{n} \rightarrow D x$ in $L^{p}\left(Z, \boldsymbol{R}^{N}\right)$ and so $x_{n} \rightarrow x$ in $W_{0}^{1, p}(Z)$ which proves the claim.

CLAIM 2. There exist $\beta_{8}, \beta_{9}>0$ such that $\phi(x) \geq \beta_{8}\|x\|^{p}-\beta_{9}\|x\|^{v}$ with $p<v \leq p^{*}$.

By virtue of hypothesis $H(f)_{1}$ (iv), given $\varepsilon>0$ we can find $\delta>0$ such that for almost all $z \in Z$ and all $|x| \leq \delta$ we have

$$
F(z, x) \leq \frac{1}{p}\left(-\lambda_{1}+\varepsilon\right)|x|^{p}
$$

Also from hypothesis $H(f)_{1}(\mathrm{ii})$, we have

$$
|F(z, x)| \leq a_{4}(z)+\eta^{\prime}|x|^{p} \quad \text { a.e. on } Z
$$

with $a_{4} \in L^{\infty}(Z), \eta^{\prime}>0$. Therefore we can find $\sigma>0$ large enough so that for $p<v \leq p^{*}$ we have 


$$
F(z, x) \leq \frac{1}{p}\left(-\lambda_{1}+\varepsilon\right)|x|^{p}+\sigma|x|^{v} \quad \text { a.e. on } Z, \text { for all } x \in \boldsymbol{R} .
$$

Therefore for every $x \in W_{0}^{1, p}(Z)$ we have

$$
\begin{aligned}
\phi(x) & =\frac{1}{p}\|D x\|_{p}^{p}-\frac{\lambda_{1}}{p}\|x\|_{p}^{p}-\int_{Z} F(z, x(z)) d z \\
& \geq \frac{1}{p}\|D x\|_{p}^{p}-\frac{\varepsilon}{\lambda_{1} p}\|D x\|_{p}^{p}-\sigma\|x\|_{v}^{v} \\
& =\frac{1}{p}\left(1-\frac{\varepsilon}{\lambda_{1}}\right)\|D x\|_{p}^{p}-\sigma\|x\|_{v}^{v} .
\end{aligned}
$$

Choose $\varepsilon>0$ so that $\varepsilon<\lambda_{1}$. So from Poincaré's inequality and since $W_{0}^{1, p}(Z)$ is embedded continuously in $L^{v}(Z)$ (recall that $v \leq p^{*}$ ), we can find $\beta_{8}, \beta_{9}>0$ such that

$$
\phi(x) \geq \beta_{8}\|x\|^{p}-\beta_{9}\|x\|^{v} \text { for all } x \in W_{0}^{1, p}(Z) .
$$

This proves the claim.

Using Claim 2 we can find $r>0$ small enough such that

$$
\inf [\phi(x):\|x\|=r]>0 .
$$

On the other hand $\phi(0)=0$ and by hypothesis $H(f)_{1}(\mathrm{v})$ for the particular $\xi \neq 0$, we have $\phi\left(\xi u_{1}\right)=\left(|\xi|^{p} / p\right)\left\|D u_{1}\right\|_{p}^{p}-\left(\lambda_{1}|\xi|^{p} / p\right)\left\|u_{1}\right\|_{p}^{p}-\int_{Z} F\left(z, \xi u_{1}(z)\right) d z=$ $\int_{Z} F\left(z, \xi u_{1}(z)\right) d z \leq 0$ (see (2)). So Claim 1 permits the use of Theorem 6 which gives us $x \in W_{0}^{1, p}(Z)$ such that

$$
\phi(x)>0 \text { (hence } x \neq 0 \text { ) and } 0 \in \partial \phi(x) .
$$

From the inclusion we have that

$$
A(x)-\lambda_{1}|x|^{p-2} x-u=0
$$

with $u \in \partial \psi(x)$, hence $f_{1}(z, x(z)) \leq u(z) \leq f_{1}(z, x(z))$ a.e. on $Z$. For every $\theta \in$ $C_{0}^{\infty}(Z)$ we have

$$
\begin{gathered}
\int_{Z}\|D x(z)\|^{p-2}(D x(z), D \theta(z))_{\boldsymbol{R}^{N}} d z \\
-\lambda_{1} \int_{Z}|x(z)|^{p-2} x(z) \theta(z) d z-\int_{Z} u(z) \theta(z) d z=0 \\
\Rightarrow\left\langle-\operatorname{div}\left(\|D x\|^{p-2} D x\right), \theta\right\rangle=\lambda_{1} \int_{Z}|x(z)|^{p-2} x(z) \theta(z) d z+\int_{Z} u(z) \theta(z) d z .
\end{gathered}
$$

From the representation theorem for the elements in $W^{-1, q}(Z)$ (see Adams [1]), we see that $\operatorname{div}\left(\|D x\|^{p-2} D x\right) \in W^{-1, q}(Z)$. Note that $C_{0}^{\infty}(Z)$ is dense in $W_{0}^{1, p}(Z)$ and $W^{-1, q}(Z)=W_{0}^{1, p}(Z)^{*}$. So from (24) it follows that 


$$
\left\{\begin{array}{l}
-\operatorname{div}\left(\|D x(z)\|^{p-2} D x(z)\right)-\lambda_{1}|x(z)|^{p-2} x(z)=f(z, x(z)) \text { a.e. on } Z \\
x_{\left.\right|_{\Gamma}}=0, \quad 2 \leq p<\infty
\end{array}\right\}
$$

and hence $x \in W_{0}^{1, p}(Z)$ is a nontrivial solution of (2). $\quad$ Q.E.D.

\section{Multiple solutions for problems at resonance}

In this section we consider quasilinear problems at resonance with Caratheodory right hand side. So we deal with problem (15). Using Theorem 1, we prove the existence of at least two nontrivial solutions. Recall that a function $f: Z \times \boldsymbol{R} \rightarrow \boldsymbol{R}$ is a Caratheodory function if for all $x \in \boldsymbol{R}, z \rightarrow f(z, x)$ is measurable and for almost all $z \in Z, x \rightarrow f(z, x)$ is continuous. Recall that a Caratheodory function is jointly measurable, hence $N$-measurable (see $\mathrm{Hu}$ Papageorgiou [12, Proposition II.1.6, p. 142]). The hypotheses on the nonlinearity $f(z, x)$ are the following:

$\boldsymbol{H}(\boldsymbol{f})_{2}: \quad f: Z \times \boldsymbol{R} \rightarrow \boldsymbol{R}$ is a Caratheodory function such that

(i) for every $M>0$, there exists $a_{M} \in L^{\infty}(Z)$ such that for almost all $z \in Z$ and all $|x| \leq M$ we have $|f(z, x)| \leq a_{M}(z)$;

(ii) there exists $\delta>0$ such that for almost all $z \in Z$ and all $|x| \leq \delta$, we have $F(z, x) \geq 0$;

(iii) there exists $\theta \in L^{\infty}(Z)$ with $\theta(z) \leq 0$ a.e. on $Z$ and the inequality is strict on a set of positive Lebesgue measure such that $\varlimsup_{|x| \rightarrow \infty} p F(z, x)=\theta(z)$ uniformly for almost all $z \in Z$;

(iv) $\lim _{x \rightarrow 0}\left(p F(z, x) /|x|^{p}\right)=0$ uniformly for almost all $z \in Z$;

(v) there exists $\xi>0$ such that $\int_{Z} F\left(z, \xi u_{1}(z)\right) d z>0$.

Remark. By virtue of hypothesis $H(f)_{2}$ (iii) the potential function $F(z, x)$ has a finite limit as $|x| \rightarrow \infty$ for almost all $z \in Z$. Hence according to the prevaling terminology the problem is "strongly resonant". Such problems for semilinear equations $(p=2)$ were studied by Bartolo-Benci-Fortunato [4] (who coined the term "strongly resonant problem"), Goncalves-Miyagaki [11], Thews [23] and Ward [25]. It should be pointed out that compared to GoncalvesMiyagaki [11] (who also have a multiplicity result) our overall hypotheses on $f$ are weaker. However, they prove the existence of three nontrivial solutions (always for the $p=2$ problem, semilinear problem). Their result was recently extended to quasilinear problems by Kourogenis-Papageorgiou [17], using of course stronger hypotheses than $\mathrm{H}(f)_{2}$.

We have the following multiplicity result:

THEOREM 11. If hypotheses $H(f)_{2}$ hold, then problem (15) has at least two nontrivial solutions. 
Proof. As before the energy functional $\phi: W_{0}^{1, p}(Z) \rightarrow \boldsymbol{R}$ is defined by

$$
\phi(x)=\frac{1}{p}\|D x\|_{p}^{p}-\frac{\lambda_{1}}{p}\|x\|_{p}^{p}-\int_{Z} F(z, x(z)) d z .
$$

Now we have $\phi \in C^{1}\left(W_{0}^{1, p}(Z)\right)$. By virtue of hypothesis $H(f)_{2}(\mathrm{iv})$, we can find $1 \geq \delta_{1}=\delta_{1}(\varepsilon)>0$ such that for almost all $z \in Z$ and all $|x| \leq \delta_{1}$ we have $F(z, x) \leq(\varepsilon / p)|x|^{p}$. Combined this with hypothesis $H(f)_{2}(\mathrm{i})$ implies that for almost all $z \in Z$ and all $x \in \boldsymbol{R}$ we have

$$
F(z, x) \leq \frac{\varepsilon}{p}|x|^{p}+\beta_{1}|x|^{\mu}
$$

with $p<\mu \leq p^{*}$ (recall that $p^{*}$, the critical Sobolev exponent, equals $N p /(N-p)$ if $p<N$ and $+\infty$ if $p \geq N)$.

Let $W_{0}^{1, p}(Z)=Y \oplus V$, where $Y=\boldsymbol{R} u_{1}$ and $V$ its topological complement.

Claim 1. There exists $r_{1}>0$ such that $\phi(v) \geq 0$ for all $v \in V,\|v\| \leq r_{1}$.

Using (3) and (25), for every $v \in V$, we have

$$
\begin{aligned}
\phi(v) & =\frac{1}{p}\|D v\|_{p}^{p}-\frac{\lambda_{1}}{p}\|v\|_{p}^{p}-\int_{Z} F(z, x(z)) d z \\
& \geq \frac{1}{p}\|D v\|_{p}^{p}-\frac{\lambda_{1}}{\lambda^{*} p}\|D v\|_{p}^{p}-\frac{\varepsilon}{p}\|v\|_{p}^{p}-\beta_{2}\|v\|_{p}^{\theta} \quad \text { for some } \beta_{2}>0 \\
& \geq \frac{1}{p}\|D v\|_{p}^{p}-\frac{\lambda_{1}}{\lambda^{*} p}\|D v\|_{p}^{p}-\frac{\varepsilon}{\lambda^{*} p}\|D v\|_{p}^{p}-\beta_{3}\|D v\|_{p}^{\theta} \quad \text { for some } \beta_{3}>0 \\
& \geq \frac{1}{p}\left(1-\frac{\lambda_{1}+\varepsilon}{\lambda^{*}}\right)\|D v\|_{p}^{p}-\beta_{3}\|D v\|_{p}^{\theta}
\end{aligned}
$$

Choose $\varepsilon>0$ so that $\lambda_{1}+\varepsilon<\lambda^{*}$. Then we have

$$
\phi(v) \geq \beta_{4}\|D v\|_{p}^{p}-\beta_{3}\|D v\|_{p}^{\theta}
$$

for some $\beta_{4}>0$ and all $v \in V$. Since $\theta>p$, by choosing $r_{1}>0$ small enough we see that $\phi(v) \geq 0$ for all $v \in V,\|v\| \leq r_{1}$.

Claim 2. There exists $r_{2}>0$ such that $\phi\left(t u_{1}\right) \leq 0$ for all $|t| \leq r_{2}$.

We have

$$
\begin{aligned}
\phi\left(t u_{1}\right) & =\frac{|t|^{p}}{p}\left\|D u_{1}\right\|_{p}^{p}-\frac{|t|^{p}}{p} \lambda_{1}\left\|u_{1}\right\|_{p}^{p}-\int_{Z} F\left(z, t u_{1}(z)\right) d z \\
& =-\int_{Z} F\left(z, t u_{1}(z)\right) d z .
\end{aligned}
$$

Since $u_{1} \in C^{1}(\bar{Z})$ (see Lieberman [19, Theorem 1]), from hypothesis $H(f)_{2}$ (ii) it follows that if $r_{2}=\delta /\left\|u_{1}\right\|_{\infty}$, we have that $\phi\left(t u_{1}\right) \leq 0$ for all $|t| \leq r_{2}$. 
ClaIm 3. $\phi(\cdot)$ satisfies the (PS)-condition.

Let $\left\{x_{n}\right\}_{n \geq 1} \subseteq W_{0}^{1, p}(Z)$ be such that $\left\{\phi\left(x_{n}\right)\right\}_{n \geq 1}$ is bounded and $\phi^{\prime}\left(x_{n}\right) \stackrel{n \rightarrow \infty}{\longrightarrow} 0$. Let $\psi: W_{0}^{1, p}(Z) \rightarrow \boldsymbol{R}$ be defined by $\psi(x)=\|D x\|_{p}^{p}-\lambda_{1}\|x\|_{p}^{p}-\int_{Z} \theta(z) d z$. We will show that there exists $\xi>0$ such that $\psi(x) \geq \xi\|D x\|_{p}^{p}$. Suppose not. Then we can find $\left\{x_{m}\right\}_{m \geq 0} \subseteq W_{0}^{1, p}(Z)$ with $\left\|D x_{m}\right\|_{p}=1$ such that $\psi\left(x_{m}\right) \downarrow 0$. Using Poincaré's inequality and by passing to a subsequence if necessary, we may assume that $x_{m} \stackrel{w}{\rightarrow} x$ in $W_{0}^{1, p}(Z)$ and $x_{m} \rightarrow x$ in $L^{p}(Z)$. Thus we have

$$
\begin{aligned}
0=\lim \psi\left(x_{m}\right) & \geq \underline{\lim }\left\|D x_{m}\right\|_{p}^{p}-\lambda_{1}\|x\|_{p}^{p}-\int_{Z} \theta(z) d z \\
& \geq\|D x\|_{p}^{p}-\lambda_{1}\|x\|_{p}^{p}-\int_{Z} \theta(z) d z>0 \quad \text { (Rayleigh quotient) }
\end{aligned}
$$

a contradiction. So there exists $\xi>0$ such that $\psi(x) \geq \xi\|D x\|_{p}^{p}$ for all $x \in$ $W^{1, p}(Z)$.

Now by virtue of hypothesis $H(f)_{2}$ (iii), given $\varepsilon>0$ we can find $M=M(\varepsilon)>0$ such that for almost all $z \in Z$ and all $|x|>M$, we have $F(z, x) \leq(1 / p) \theta(z)$. On the other hand, from hypothesis $H(f)_{2}(\mathrm{i})$, we know that for almost all $z \in Z$ and all $|x| \leq M$, we have $|F(z, x)| \leq a_{M}(z)$. Thus we infer that there exists $a_{1} \in L^{\infty}(Z)$ (take for example $a_{1}(z)=a_{M}(z)+\|\theta\|_{\infty}$ ) such that for almost all $z \in Z$ and all $z \in \boldsymbol{R}$, we have

$$
F(z, x) \leq \theta(z)+\varepsilon+a_{1}(z)
$$

Using (26) we have

$$
\begin{aligned}
\phi(x) & \geq \frac{1}{p}\|D x\|_{p}^{p}-\frac{\lambda_{1}}{p}\|x\|_{p}^{p}+\frac{1}{p} \int_{Z} \theta(z) d z-\beta_{5}, \text { for some } \beta_{5}>0 \\
& \geq \frac{1}{p} \xi\|D x\|_{p}^{p}-\beta_{5} .
\end{aligned}
$$

From the above inequality we see that $\phi(\cdot)$ is coercive. Since $\left\{\phi\left(x_{n}\right)\right\}_{n \geq 1}$ is bounded, we must have that $\left\{x_{n}\right\}_{n \geq 1} \subseteq W_{0}^{1, p}(Z)$ is bounded and so by passing to a subsequence if necessary, we may assume that $x_{n} \stackrel{w}{\rightarrow} x$ in $W_{0}^{1, p}(Z)$. Then arguing as in the proof of Theorem 10 we can have that $x_{n} \rightarrow x$ in $W_{0}^{1, p}(Z)$, which proves the claim.

Finally note that $\phi(\cdot)$ being coercive is bounded below, while by virtue of hypothesis $H(f)_{2}(\mathrm{v})$ and since $\left\|D u_{1}\right\|_{p}^{p}=\lambda_{1}\left\|u_{1}\right\|_{p}^{p}$, we have that $\inf _{W_{0}^{1, p}(Z)} \phi<0$. These facts together with Claims 1, 2 and 3, allow as to use Theorem 1, which gives $x_{1} \neq x_{2}, x_{1}, x_{2} \neq 0$, such that $\phi^{\prime}\left(x_{1}\right)=\phi^{\prime}\left(x_{2}\right)=0$. The same argument as in the proof of Theorem 10, shows that $x_{1}, x_{2} \in W_{0}^{1, p}(Z)$ are nontrivial distinct solutions of (15). 


\section{Semilinear problems at resonance}

In this section we prove an existence theorem for the semilinear problem (i.e., $p=2$ ) at resonance with a discontinuous right hand side. So our problem is the following:

$$
\left\{\begin{array}{l}
-\Delta x(z)-\lambda_{1} x(z)=f(z, x(z)) \text { a.e. on } Z \\
x_{\left.\right|_{\Gamma}}=0 .
\end{array}\right\}
$$

As before (see section 4), since we do not require $f(z, \cdot)$ to be continuous, by introducing the functions $f_{1}(z, x)=\varliminf_{x^{\prime} \rightarrow x} f\left(z, x^{\prime}\right)$ and $f_{2}(z, x)=\varlimsup_{x^{\prime} \rightarrow x} f\left(z, x^{\prime}\right)$, we pass to the following multivalued approximation of (27):

$$
\left\{\begin{array}{l}
-\Delta x(z)-\lambda_{1} x(z) \in \hat{f}(z, x(z)) \text { a.e. on } Z \\
x_{\left.\right|_{\Gamma}}=0 .
\end{array}\right\}
$$

where $\hat{f}(z, x)=\left\{y \in \boldsymbol{R}: f_{1}(z, x) \leq y \leq f_{2}(z, x)\right\}$. Our hypotheses on the discontinuous nonlinearity $f(z, x)$ are the following:

$\boldsymbol{H}(\boldsymbol{f})_{3}: \quad f: Z \times \boldsymbol{R} \rightarrow \boldsymbol{R}$ is a Borel measurable function such that

(i) $f_{1}$ and $f_{2}$ are both $N$-measurable functions

(ii) for every $M>0$, there exists $a_{M} \in L^{2}(Z)$ such that for almost all $z \in Z$ and all $|x| \leq M$ we have $|f(z, x)| \leq a_{M}(z)$;

(iii) $\lim _{|x| \rightarrow \infty} f(z, x) / x=0$ uniformly for almost all $z \in Z$;

(iv) if

$$
G_{1}(z, x)= \begin{cases}\frac{2}{x} \int_{0}^{x} f(z, x) d r-f_{1}(z, x) & \text { if } x \neq 0 \\ 0 & \text { if } x=0\end{cases}
$$

and

$$
G_{2}(z, x)= \begin{cases}\frac{2}{x} \int_{0}^{x} f(z, x) d r-f_{2}(z, x) & \text { if } x \neq 0 \\ 0 & \text { if } x=0\end{cases}
$$

then $G_{1}^{-}(z)=\lim _{x \rightarrow-\infty} G_{1}(z, x)$ and $G_{2}^{+}(z)=\lim _{x \rightarrow+\infty} G_{2}(z, x)$ exist uniformly for almost all $z \in Z, G_{1}^{-}, G_{2}^{+} \in L^{2}(Z)$ and $\int_{Z} G_{1}^{-}(z) u_{1}(z) d z<0<\int_{Z} G_{2}^{+}(z) u_{1}(z) d z$.

Remark. Hypothesis $H(f)_{3}$ (iv) generalizes the well-known LandesmanLazer conditions, even in the continuous case (i.e., $f(z, \cdot)$ continuous). Thus, in this respect our theorem extends the results of Solimini [22] (see also Ghoussoub [9, Theorems 9.14 and 9.16]). Another generalization of the Landesman-Lazer condition can be found in the work of Landesman-Robinson-Rumbos [18].

We have the following existence theorem: 
THEOREM 12. If hypotheses $H(f)_{3}$ hold, then problem (28) has at least one nontrivial solution.

Proof. We consider the energy functional $\phi: H_{0}^{1}(Z) \rightarrow \boldsymbol{R}$ defined by

$$
\phi(x)=\frac{1}{2}\|D x\|_{2}^{2}-\frac{\lambda_{1}}{2}\|x\|_{2}^{2}-\int_{Z} F(z, x(z)) d z .
$$

By virtue of hypothesis $H(f)_{3}$ (iii), given $\varepsilon>0$ there exists $M=M(\varepsilon)>0$ such that for almost all $z \in Z$ and all $|x|>M$ we have $|f(z, x)| \leq \varepsilon|x|$. Combining this with hypothesis $H(f)_{3}$ (ii) we infer that for almost all $z \in Z$ and all $x \in \boldsymbol{R}$, we have

$$
|f(z, x)| \leq \varepsilon|x|+a_{1}(z)
$$

with $a_{1} \in L^{2}(Z)$. Evidently the same growth condition is satisfied by $f_{1}$ and $f_{2}$.

Claim 1. The energy functional $\phi(\cdot)$ satisfies the nonsmooth PS-condition.

Let $\left\{x_{n}\right\}_{n \geq 1} \subseteq H_{0}^{1}(z)$ be a sequence such that $\left\{\phi\left(x_{n}\right)\right\}_{n \geq 1}$ is bounded and $m\left(x_{n}\right) \stackrel{n \rightarrow \infty}{\longrightarrow} 0$. We will show that $\left\{x_{n}\right\}_{n \geq 1}$ is bounded. Suppose not. Then we may assume that $\left\|x_{n}\right\| \stackrel{n \rightarrow \infty}{\longrightarrow} \infty$. Let $x_{n}^{*} \in \partial \phi\left(x_{n}\right), n \geq 1$, such that $m\left(x_{n}\right)=\left\|x_{n}^{*}\right\|$. We have

$$
x_{n}^{*}=A\left(x_{n}\right)-\lambda_{1} x_{n}-w_{n}, \quad n \geq 1,
$$

where $A \in \mathscr{L}\left(H_{0}^{1}(Z), H^{-1}(Z)\right)$ is defined by $\langle A(x), y\rangle=\int_{Z}(D x(z), D y(z))_{R^{N}} d z$ and $w_{n} \in L^{2}(Z), f_{1}\left(z, x_{n}(z)\right) \leq w_{n}(z) \leq f_{2}\left(z, x_{n}(z)\right)$ a.e. on $Z$. Let $Y=\boldsymbol{R} u_{1}$ and $V=Y^{\perp}$. Then $H_{0}^{1}(Z)=Y \oplus V$. We can write that $x_{n}=t_{n} u_{1}+v_{n}$, with $t_{n} \in \boldsymbol{R}$ and $v_{n} \in V$. From the choice of the sequence $\left\{x_{n}\right\}_{n \geq 1}$

$$
\begin{aligned}
& \left|\left\langle x_{n}^{*}, v_{n}\right\rangle\right| \leq \beta_{1}\left\|v_{n}\right\| \text { for some } \beta_{1}>0 \\
\Rightarrow & \left\|D v_{n}\right\|_{2}^{2}-\lambda_{1}\left\|v_{n}\right\|_{2}^{2}-\int_{Z} w_{n}(z) v_{n}(z) d z \leq \beta_{1}\left\|v_{n}\right\|_{1,2} \\
& \left(\text { since } \int_{Z} u_{1}(z) v_{n}(z) d z=0\right) \\
\Rightarrow & \left\|D v_{n}\right\|_{2}^{2}-\lambda_{1}\left\|v_{n}\right\|_{2}^{2}-\varepsilon\left\|x_{n}\right\|_{2}\left\|v_{n}\right\|_{2}-\left\|a_{1}\right\|_{2}\left\|v_{n}\right\|_{2} \leq \beta_{1}\left\|v_{n}\right\|_{1,2} \text { (using (31)) } \\
\Rightarrow & \left(1-\frac{\lambda_{1}}{\lambda_{2}}\right)\left\|D v_{n}\right\|_{2}^{2}-\varepsilon\left\|x_{n}\right\|_{1,2}\left\|v_{n}\right\|_{1,2}-\left\|a_{2}\right\|_{2}\left\|v_{n}\right\|_{1,2} \leq \beta_{1}\left\|v_{n}\right\|_{1,2}
\end{aligned}
$$

(since on $V$ we have $\|D v\|_{2}^{2} \geq \lambda_{2}\|v\|_{2}^{2}$ for all $v \in V$; here $\lambda_{2}>0$ is the second eigenvalue of $\left(-\Delta, H_{0}^{1}(Z)\right)$ and $\lambda_{2}=\lambda^{*}$ (see (3))). Divide (30) with $\left\|v_{n}\right\|_{1,2}$ and using Poincaré's inequality to obtain

$$
c\left(1-\frac{\lambda_{1}}{\lambda_{2}}\right)\left\|v_{n}\right\|_{1,2}-\varepsilon\left\|x_{n}\right\|_{1,2}-\left\|a_{2}\right\|_{2} \leq \beta_{1}
$$


with $c>0$. Now divide this last inequality with $\left\|x_{n}\right\|_{1,2}$. We obtain

$$
c\left(1-\frac{\lambda_{1}}{\lambda_{2}}\right) \frac{\left\|v_{n}\right\|_{1,2}}{\left\|x_{n}\right\|_{1,2}}-\varepsilon-\frac{\|a\|_{2}}{\left\|x_{n}\right\|_{1,2}} \leq \frac{\beta_{1}}{\left\|x_{n}\right\|_{1,2}} .
$$

Since $\lambda_{1}<\lambda_{2}$, we infer that

$$
\varlimsup \frac{\left\|v_{n}\right\|_{1,2}}{\left\|x_{n}\right\|_{1,2}} \leq \frac{\lambda_{2}}{c\left(\lambda_{2}-\lambda_{1}\right)} \varepsilon
$$

Let $\varepsilon \downarrow 0$ to conclude that

$$
\frac{\left\|v_{n}\right\|_{1,2}}{\left\|x_{n}\right\|_{1,2}} \stackrel{n \rightarrow \infty}{\longrightarrow} 0
$$

Also we know that $\left\|x_{n}\right\|_{1,2}^{2}=t_{n}^{2}\left\|u_{1}\right\|_{1,2}^{2}+\left\|v_{n}\right\|_{1,2}^{2}=t_{n}^{2}+\left\|v_{n}\right\|_{1,2}^{2}$ and so

$$
\begin{aligned}
& \frac{t_{n}^{2}}{\left\|x_{n}\right\|_{1,2}^{2}}+\frac{\left\|v_{n}\right\|_{1,2}^{2}}{\left\|x_{n}\right\|_{1,2}^{2}}=1 \\
\Rightarrow & \frac{t_{n}}{\left\|x_{n}\right\|_{1,2}} \stackrel{n \rightarrow \infty}{\longrightarrow} \pm 1 .
\end{aligned}
$$

Suppose without any loss of generality that $\left(t_{n} /\left\|x_{n}\right\|_{1,2}\right) \stackrel{n \rightarrow \infty}{\longrightarrow}+1$ (the analysis is the same if $\left.\left(t_{n} /\left\|x_{n}\right\|_{1,2}\right) \stackrel{n \rightarrow \infty}{\longrightarrow}-1\right)$. Then $t_{n} \stackrel{n \rightarrow \infty}{\longrightarrow}+\infty$ and if $y_{n}=x_{n} /\left\|x_{n}\right\|_{1,2}$ we have $y_{n} \stackrel{n \rightarrow \infty}{\longrightarrow} u_{1}$ in $H_{0}^{1}(Z)$. Let

$$
h_{n}(z)=\left\{\begin{array}{ll}
F\left(z, x_{n}(z)\right) /\left(x_{n}(z)\right) & \text { if } x_{n}(z) \neq 0 \\
0 & \text { otherwise }
\end{array}, \quad n \geq 1 .\right.
$$

Then we have:

$$
\begin{aligned}
\left\langle x_{n}^{*}, y_{n}\right\rangle & -\frac{2 \phi\left(x_{n}\right)}{\left\|x_{n}\right\|_{1,2}} \\
= & \int_{Z} 2 h_{n}(z) y_{n}(z) d z-\int_{Z} w_{n}(z) y_{n}(z) d z \\
\geq & \int_{Z} 2 h_{n}(z) y_{n}(z) d z-\int_{\left\{y_{n}>0\right\}} f_{2}\left(z, x_{n}(z)\right) y_{n}(z) d z \\
& -\int_{\left\{y_{n}<0\right\}} f_{1}\left(z, x_{n}(z)\right) y_{n}(z) d z \\
= & \int_{\left\{y_{n}>0\right\}} G_{2}\left(z, x_{n}(z)\right) y_{n}(z) d z-\int_{\left\{y_{n}<0\right\}} G_{1}\left(z, x_{n}(z)\right) y_{n}(z) d z
\end{aligned}
$$

From the choice of the sequence $\left\{x_{n}\right\}_{n \geq 1} \subseteq H_{0}^{1}(Z)$ we have that $\left\langle x_{n}^{*}, y_{n}\right\rangle$ $\stackrel{n \rightarrow \infty}{\longrightarrow} 0$ and $2 \phi\left(x_{n}\right) /\left\|x_{n}\right\|_{1,2} \leq M /\left\|x_{n}\right\|_{1,2} \stackrel{n \rightarrow \infty}{\longrightarrow} 0$. In addition, at least for a 
subsequence, we have $\chi_{\left\{y_{n}>0\right\}} \stackrel{n \rightarrow \infty}{\longrightarrow} \chi_{Z}=1$ a.e. on $Z$. Thus by passing to the limit in (32), we obtain

$$
\int_{Z} G_{2}^{+}(z) u_{1}(z) d z \leq 0
$$

which contradicts hypothesis $H(f)_{3}$ (iv). This proves the boundedness of $\left\{x_{n}\right\}_{n \geq 1}$ $\subseteq H_{0}^{1}(Z)$. So we may assume that $x_{n} \underset{n \rightarrow \infty}{\stackrel{w}{\longrightarrow}} x$ in $H_{0}^{1}(Z)$ as $n \rightarrow \infty$ and proceeding as in previous proofs, we have that $x_{n} \stackrel{n \rightarrow \infty}{\longrightarrow} x$ in $H_{0}^{1}(Z)$ and so $\phi(\cdot)$ satisfies the nonsmooth PS-condition.

Claim 2. $\phi\left(t u_{1}\right) \rightarrow-\infty$ as $|t| \rightarrow \infty$.

From hypothesis $H(f)_{3}$ (iv), given $\varepsilon>0$, we can find $M=M(\varepsilon)>0$ such that for almost all $z \in Z$ and all $x \leq-M$ we have

$$
\begin{aligned}
G_{1}(z, x) & \leq G_{1}^{-}(z)+\varepsilon=\theta_{\varepsilon}^{1}(z) \\
\Rightarrow \quad \frac{G_{1}(z, x)}{x^{2}} & \leq \frac{\theta_{\varepsilon}^{1}(z)}{x^{2}}=\frac{d}{d x}\left(-\frac{\theta_{\varepsilon}^{1}(z)}{x}\right) .
\end{aligned}
$$

Also note that from the definition of $G_{1}(z, x)$, we have

$$
\begin{aligned}
\frac{G_{1}(z, x)}{x^{2}} & \geq \frac{1}{x^{2}}\left(\frac{2}{x} \int_{0}^{x} f(z, r) d r-f(z, x)\right) \\
& =\frac{2}{x^{3}} \int_{0}^{x} f(z, r) d r-\frac{1}{x^{2}} f(z, x) \\
& =\frac{d}{d x}\left(-\frac{1}{x^{2}} \int_{0}^{x} f(z, r) d r\right)=\frac{d}{d x}\left(-\frac{F(z, x)}{x^{2}}\right) .
\end{aligned}
$$

Using this inequality in (33), we obtain

$$
\frac{d}{d x}\left(-\frac{F(z, x)}{x^{2}}\right) \leq \frac{d}{d x}\left(-\frac{\theta_{\varepsilon}^{1}(z)}{x}\right) \text { a.e. on } Z \text {, for a.a. } x \leq-M
$$

Integrating this inequality on $[y, x], y<x \leq-M$, we have

$$
-\frac{F(z, x)}{x^{2}}+\frac{F(z, y)}{y^{2}} \leq \frac{-\theta_{\varepsilon}^{1}(z)}{x}+\frac{\theta_{\varepsilon}^{1}(z)}{y} .
$$

From (29) we know that for almost all $z \in Z$ and all $r \leq 0$ we have

$$
\begin{aligned}
& f(z, x) \leq-\varepsilon r+a_{1}(z), a_{1} \in L^{2}(Z), \\
\Rightarrow & \frac{1}{y^{2}} \int_{0}^{y} f(z, r) d r \geq-\frac{\varepsilon}{2}+\frac{a_{1}(z)}{y^{2}} \\
\Rightarrow & \varliminf_{y \rightarrow-\infty} \frac{F(z, y)}{y^{2}} \geq-\frac{\varepsilon}{2} .
\end{aligned}
$$


Let $\varepsilon \downarrow 0$ to conclude that

$$
\varliminf_{y \rightarrow-\infty} \frac{F(z, y)}{y^{2}} \geq 0 .
$$

So, if in (34) we let $y \rightarrow-\infty$, we obtain that for almost all $z \in A$ and all $x \leq-M$ we have

$$
\begin{aligned}
& \frac{F(z, x)}{x} \leq \theta_{\varepsilon}^{1}(z) \\
\Rightarrow & \varlimsup_{x \rightarrow-\infty} \frac{F(z, x)}{x} \leq \theta_{\varepsilon}^{1}(z) \text { a.e. on } Z .
\end{aligned}
$$

Letting $\varepsilon \downarrow 0$, we have that

$$
\varlimsup_{x \rightarrow-\infty} \frac{F(z, x)}{x} \leq G_{1}^{-}(z) \text { a.e. on } Z .
$$

Similarly we can show that

$$
\varliminf_{x \rightarrow+\infty} \frac{F(z, x)}{x} \geq G_{2}^{+}(z) \text { a.e. on } Z \text {. }
$$

Suppose the claim was not true. Then we can find $\left|t_{n}\right| \rightarrow+\infty$ such that $\phi\left(t_{n} u_{1}\right) \geq-\gamma$ for some $\gamma>0$. First assume that $t_{n} \rightarrow-\infty$. We have $\left(1 / t_{n}\right) \phi\left(t_{n} u_{1}\right) \leq-\gamma / t_{n}$. Therefore

$$
\begin{aligned}
& \varlimsup \frac{1}{t_{n}} \phi\left(t_{n} u_{1}\right) \leq 0 \\
\Rightarrow & \varlimsup \\
\Rightarrow & \underline{\lim }-\frac{1}{t_{n}} \int_{Z} F\left(z, t_{n} u_{1}(z)\right) d z \leq 0 \\
\Rightarrow & \int_{Z} G_{1}^{-}(z) u_{1}(z) d z \geq 0, \quad(\operatorname{see}(35))
\end{aligned}
$$

which contradicts $H(f)_{3}$ (iv). Similarly if $t_{n} \rightarrow+\infty$, we obtain using (36)

$$
\int_{Z} G_{2}^{+}(z) u_{1}(z) d z \leq 0
$$

which contradicts $H(f)_{3}$ (iv). Therefore the claim is true and we have $\phi\left(t u_{1}\right) \stackrel{|t| \rightarrow \infty}{\longrightarrow}-\infty$.

ClaIm 3. $\phi(v) \rightarrow+\infty$ as $\|v\|_{1,2} \rightarrow \infty, v \in V$ (hence $\phi_{\left.\right|_{V}}$ is bounded below).

Since for $v \in V,\|D v\|_{2}^{2} \geq \lambda_{2}\|v\|_{2}^{2}, \lambda_{2}>\lambda_{1}$ and using (29) we have for all 
$v \in V$

$$
\begin{aligned}
\phi(v) & \geq \frac{1}{2}\|D v\|_{2}^{2}-\frac{\lambda_{1}}{2}\|v\|_{2}^{2}-\frac{\varepsilon}{2}\|v\|_{2}^{2}-\left\|a_{1}\right\|_{2}\|v\|_{2} \\
& \geq \frac{1}{2}\left(1-\frac{\lambda_{1}}{\lambda_{2}}-\frac{\varepsilon}{\lambda_{2}}\right)\|D v\|_{2}^{2}-\beta\|D v\|_{2} \text { for some } \beta>0
\end{aligned}
$$

Choose $\varepsilon>0$ so that $\lambda_{1}+\varepsilon<\lambda_{2}$. Then from the above inequality, it is clear that $\phi(v) \rightarrow \infty$ as $\|v\|_{1,2} \rightarrow \infty$. Hence $\phi_{\left.\right|_{V}}$ is bounded below.

Claims 1, 2 and 3 permit the application of Theorem 7, which gives us $x \in H_{0}^{1}(Z)$ such that $0 \in \partial \phi(x)$. As before we conclude that $x$ solves $(30)$.

Q.E.D.

Remark. We know that in this case there is an orthonormal basis $\left\{u_{m}\right\}_{m \geq 1}$ of $L^{2}(Z)$ and a sequence of positive real numbers $\left\{\lambda_{m}\right\}_{m \geq 1}$ with $\lambda_{m} \rightarrow+\infty$ such that $0<\lambda_{1}<\lambda_{2} \leq \lambda_{3} \leq \cdots \leq \lambda_{m} \leq \cdots$ and $u_{m} \in H_{0}^{1}(Z) \cap C^{\infty}(Z), m \geq 1$, are solutions of (1) with $p=2$. Moreover, these higher eigenvalues have variational characterizations similar to (2) (see Kesavan [13]). So, in this case, in contrast to the case $p>2$, we have full knowledge of the spectrum of $\left(-\Delta, H_{0}^{1}(Z)\right)$. Thus what we did for the resonant at $\lambda_{1}$ problem, we can do it for the problem which is resonant at some higher eigenvalue, using the same approach with minor modifications.

Acknowledgement. The authors wish to express their gratitude to a very knowledgeable referee for his corrections and remarks that improved the paper considerably.

\section{REFERENCES}

[1] Adams, R., Sobolev Spaces, Academic Press, New York, 1975.

[2] Ahmad, S., Multiple nontrivial solutions of resonant and nonresonant asymptotically linear problems, Proc. Amer. Math. Soc., 96 (1986), pp. 405-409.

[3] Ahmad, S., Lazer, A. AND Paul, J., Elementary critical point theory and perturbations of elliptıc boundary value problems at resonance, Indiana Unıv. Math. J., 25 (1976), pp. $933-$ 944.

[4] Bartolo, P., Benci, V and Fortunato, D., Abstract critical point theorems and applications to some nonlinear problems with "strong resonance" at infinity, Nonlinear Appl., 9 (1983), pp. 981-1012.

[ 5 ] Brezis, H. and Nirenberg, L., Remarks on finding critıcal points, Comm. Pure Appl. Math., 64 (1991), pp. 939-963.

[6] Cerami, G., Un criterio di esıstenza per 1 puntı critıcı su varieta illimitate, Rend. Instituto Lombardo Sc1. Lett., 112 (1978) pp. 332-336.

[7] ChANG, K.-C., Variational methods for non-differentiable functionals and their applications to partial differential equations, J. Math. Anal. Appl., 80 (1981), pp. 102-129.

[8] Clarke, F H., Optımization and Nonsmooth Analysıs, Wiley, New York, 1983.

[9] Ghoussoub, N., Duality and Perurbation Methods in Critical Point Theory, Cambridge University Press, Cambridge, 1993. 
[10] Goncalves, J. AND MiYagaki, O., Multıple nontrivial solutions of semilinear strongly resonant elliptic equations, Nonlinear Anal., 19 (1992), pp. 43-52.

[11] Goncalves, J. AND MiYagaki, O., Three solutions for a strongly resonant elliptic problems, Nonlinear Anal., 24 (1995), pp. 265-272.

[12] Hu, S. and Papageorgiou, N. S., Handbook of Multivalued Analysis, Volume I: Theory, Kluwer, Dordrecht, 1997

[13] Kesavan, S., Topics in Functional Analysis and Applications, Wiley, New York, 1989.

[14] Kourogenis, N. C. and Papageorgiou, N. S., Discontinuous quasilinear elliptic problems at resonance, Colloq. Math., 78 (1998), pp. 213-223.

[15] Kourogenis, N. C. And Papageorgiou, N. S., Multiple solutions for nonlinear discontınuous elliptıc equations near resonance, Colloq. Math., 81 (1999), pp. 80-99.

[16] Kourogenis, N. C. And Papageorgiou, N. S., Three nontrivial solutions for a quasilinear elliptıc differentıal equation at resonance with discontınuous right hand side, J. Math. Anal. Appl., 238 (1999), pp. 477-490.

[17] Kourogenis, N. C. and Papageorgiou, N. S., Multıple solutıons for nonlinear discontınuous strongly resonant problems, to appear in J. Math. Soc. Japan.

[18] Landesman, E., Robinson, S. and Rumbos, A., Multiple solutions of semilinear elliptic problems at resonance, Nonlinear Anal., 24 (1995), pp. 1049-1059.

[19] Lieberman, G., Boundary regularity for solutions of degenerate elliptic equations, Nonlinear Anal., 12 (1988), pp. 1203-1219.

[20] Lindevist, P., On the equation $\operatorname{div}\left(\|D x\|^{p-2} D x\right)+\lambda|x|^{p-2} x=0$, Proc. Amer. Math. Soc., 109 (1990), pp. 157-164.

[21] Rabinowitz, P., Minımax Methods in Critıcal Point Theory with Applications to Differential Equations, CBMS Regional Conf. Ser. in Math., 65, Amer. Math. Soc., Providence, R. I., 1986.

[22] Solimini, S., On the solvability of some elliptıc partial differentıal equations with linear part at resonance, J. Math. Anal. Appl., 117 (1986), pp. 138-152.

[23] Thews, K., Nontrivial solutions of elliptic equations at resonance, Proc. Royal Soc. Edinburgh Sect. A, 85 (1990), pp. 119-129.

[24] Tolksdorf, P., Regularity for a more general class of quasilinear elliptıc equatıons, J. Differential Equations, 51 (1984), pp. 126-150.

[25] WARD, J., Applications of critical point theory to weakly nonlinear boundary value problems at resonance, Houston J. Math., 10 (1984), pp. 291-305.

[26] ZhONG, C.-K., On Ekeland's variational principle and a minımax theorem, J. Math. Anal. Appl., 205 (1997), pp. 239-250.

\author{
National Technical University \\ Department of Mathematics \\ Zografou CAMPUS \\ Athens 15780, GreECE \\ e-mail: npapg@math.ntua.gr
}

